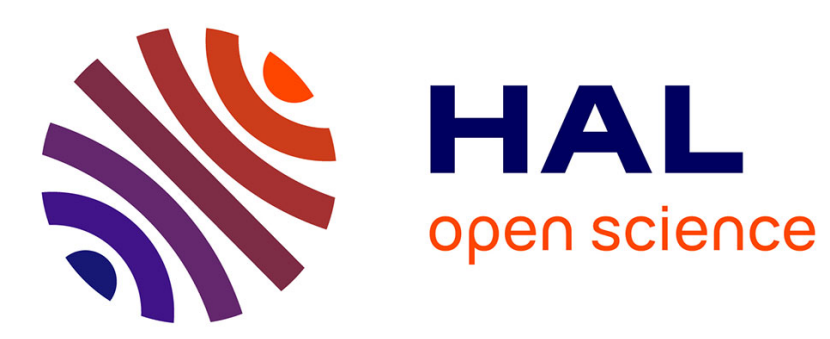

\title{
Locomotion of articulated bodies in an ideal fluid: 2d model with buoyancy, circulation and collisions
}

Bruno Pinçon, Alexandre Munnier

\section{To cite this version:}

Bruno Pinçon, Alexandre Munnier. Locomotion of articulated bodies in an ideal fluid: $2 \mathrm{~d}$ model with buoyancy, circulation and collisions. 2009. hal-00394744v1

\section{HAL Id: hal-00394744 \\ https://hal.science/hal-00394744v1}

Preprint submitted on 12 Jun 2009 (v1), last revised 11 Jan 2010 (v2)

HAL is a multi-disciplinary open access archive for the deposit and dissemination of scientific research documents, whether they are published or not. The documents may come from teaching and research institutions in France or abroad, or from public or private research centers.
L'archive ouverte pluridisciplinaire HAL, est destinée au dépôt et à la diffusion de documents scientifiques de niveau recherche, publiés ou non, émanant des établissements d'enseignement et de recherche français ou étrangers, des laboratoires publics ou privés. 


\title{
Locomotion of articulated bodies in an ideal fluid: $2 \mathrm{~d}$ model with buoyancy, circulation and collisions
}

\author{
Bruno Pinçon* $\quad$ Alexandre Munnier ${ }^{\dagger}$
}

June 12, 2009

\begin{abstract}
Articulated solid bodies are shape-changing bodies made of rigid solids linked together by means of holonomic constraints prescribed as functions of time. In this paper we study the locomotion of such swimming devices in an ideal fluid. Our study ranges over a wide class of problems: any number of immersed bodies are involved (without being hydrodynamically decoupled), the system fluid-bodies can be partially of totally confined and circulation, buoyancy and collisions between bodies are taken into account. We determine the Euler-Lagrange equation governing the dynamic of the system, study its well-posedness and describe a numerical scheme used in a Matlab toolbox (Biohydrodynamics Toolbox) that has been designed to realize easily related numerical simulations.
\end{abstract}

\section{Introduction}

In the last decade, serious efforts have been done by mathematicians to better understand the dynamics of swimming in a fluid. This interest has grown from the observation that fishes and aquatic mammals evolved swimming capabilities far superior to what has been achieved by naval technology and thereby that they provide an attractive model for designing biomimetic robots. Such swimming devices propelling and steering by shape-changes would be more efficient, stealthier and more maneuverable than if endowed with propellers. This explains why, for instance, autonomous underwater vehicles are catching the attention of petroleum industry for their possible use in the maintenance of offshore installations. In the area of nano-technologies, the design of nano-robots able to perform basic tasks is a challenge for the forthcoming years.

\footnotetext{
*Institut Elie Cartan UMR 7502, Nancy-Université, CNRS, INRIA, B.P. 239, F-54506 Vandoeuvre-lès-Nancy Cedex, France, bruno.pincon@iecn.u-nancy.fr, INRIA Lorraine, Projet CORIDA.

†Institut Elie Cartan UMR 7502, Nancy-Université, CNRS, INRIA, B.P. 239, F-54506 Vandoeuvre-lès-Nancy Cedex, France, Alexandre.munnier@iecn.u-nancy.fr, INRIA Lorraine, Projet CORIDA.
} 
Significant contributions to the understanding of the biomechanics of swimming are du to Lighthill [18, Taylor [30, 31] Childress 6] and $\mathrm{Wu}$ [33. We mention also an interesting survey on the general theme of fish locomotion written by Sparenberg [29].

Experiments have shown that the vortices generated by the tail of fishes play a crucial role to understand their locomotion and some models incorporate artificially vortices [21, 34, 32. If we do not neglect the viscosity effects, the relevant model consists in the non stationary Navier-Stokes equations for the fluid coupled with Newton's laws for the fish-like swimming object. This perspective is the one adopted by Carling, Williams and Bowtell in [5], Liu and Kawachi in [20], Galdi in [8] or San Martín, Scheid, Takahashi and Tucsnak in [27].

Nevertheless, among numerous mathematical articles studying fish locomotion, most of them address the case of a potential flow which is by definition vortex-free like in the works of Kelly and Murray 14, Kozlov and Onishchenko [15., Kanso, Marsden, Rowley and Melli-Huber [13], Melli, Rowley and Rufat 22. and Munnier 26. It is also the point of view we have chosen in what follows and on which we focus from now on.

Thus, in this paper we are interested in studying the dynamics of articulated solid bodies submerged in an ideal fluid. Articulated bodies are composed of rigid solids linked together by means of holonomic constraints and can be seen as a particular case of the more general concept of shape-changing bodies as it has been described in [26]. The holonomic constraints govern the relative position of the rigid parts composing the articulated bodies. They are prescribed as functions of time and stand in our model for the controls by which the swimming bodies propel and steer themselves.

It has been a standard method since the work of Thomson, Tait and Kirchhoff (summarized in the book of Lamb [17]) to invoke the least action principle of Lagrangian Mechanics for determining the Euler-Lagrange equations of motion when dealing with a problem of interactions between a perfect fluid and rigid immersed structures. Although we have proved in [25] that Newton's laws actually lead to the same result, we adopt also this approach in this work.

A central concept in such problems is the notion of mass matrix. Using classical notation we denote by $\mathbf{q}$ and $\dot{\mathbf{q}}$ the generalized coordinates and velocities; they parameterize all of the degrees of freedom of the system and they allow to write the Lagrangian of the system fluid-submerged bodies in the short form:

$$
L:=\frac{1}{2} \dot{\mathbf{q}}^{\top} \mathbb{M}(\mathbf{q}) \dot{\mathbf{q}}-P(\mathbf{q}),
$$

where the elements of the matrix $\mathbb{M}(\mathbf{q})$ (precisely the mass matrix) are homogeneous to masses and where $P(\mathbf{q})$ stands for the overall potential energy.

The Euler-Lagrange equations driving the free evolution of $\mathbf{q}$ with respect to time are ODEs (ordinary differential equations) resulting from the application to $L$ of the least action principle:

$$
\frac{d}{d t} \frac{\partial L}{\partial \dot{\mathbf{q}}}-\frac{\partial L}{\partial \mathbf{q}}=0, \quad(t \geq 0)
$$


Some of the degrees of freedom can be prescribed as functions of the others and of time. This idea writes:

$$
\mathbf{q}=F(t, \mathbf{p}),
$$

where $\mathbf{p}$ stands for the remaining degrees of freedom of our system and $F$ is a given function. Such constraints are termed holonomic and well suited to describe the kinematic of swimming robots made of rigid links. Considering now the Lagrangian as a function of $t, \mathbf{p}$ and $\dot{\mathbf{p}}$, the equations of motion for the swimming articulated bodies whose shape-changes are driven by relation 1.2 are:

$$
\frac{d}{d t} \frac{\partial L}{\partial \dot{\mathbf{p}}}-\frac{\partial L}{\partial \mathbf{p}}=0, \quad(t \geq 0) .
$$

Next, the game consists in expanding the left hand side of this equality and turn the equation into a convenient form allowing to study the locomotion induced by the shape-changes. The tricky point is the computation of the derivative of the mass matrix with respect to $\mathbf{p}$ (or more generally with respect to $\mathbf{q}$ ) because this task actually requires to differentiate the potential of the fluid with respect to the positions of the solids (parameterized by $\mathbf{q}$ ). Indeed, the potential of the fluid is defined as being the solution of a boundary value problem set in the fluid domain which depends on the positions of the solids and therefore this differentiation process leads to the computation of so-called shape derivatives.

Nevertheless this difficulty can be avoided. It is the case when the mass matrix does not depend on $\mathbf{p}$ what means that all of the positions and directions in the fluid are equivalent as seen by an observer attached to the bodies. This isotropy (or symmetry) arises when the following assumptions are fulfilled:

(H1) There is only one body in the fluid.

(H2) The overall system body-fluid fills the whole space (identified with $\mathbb{R}^{2}$ ).

(H3) The body is neutrally buoyant.

(H4) The system body-fluid is at rest at the time $t=0$.

Hypothesis (H1) can equivalently be replaced by:

$\left(\mathrm{H} 1^{\prime}\right)$ The dynamics of the immersed bodies are hydrodynamically decoupled,

which means that each body is handled as if being alone in the fluid. This approximation can be relevant when the immersed bodies are far apart but is not any longer when they are close. Most of the studies on swimming shapechanging bodies in an ideal fluid are carried out under hypotheses (H1-4) or $\left(\mathrm{H} 1^{\prime}-4\right)$.

One of our aims in this paper is to study the dynamics of articulated bodies relaxing all of these hypotheses. Thus, in a first section we address the case of $n$ rigid solids evolving freely in a fluid with potential flow, the system fluidsolids being either unbounded, either partially or totally confined and taking 
into account their buoyancy. We compute the Lagrangian, expand the EulerLagrange equation (1.1) and end up with a second order ODEs in $\mathbf{q}$ that writes:

$$
\mathbb{M} \ddot{\mathbf{q}}+\langle\Gamma(\mathbf{q}), \dot{\mathbf{q}}, \dot{\mathbf{q}}\rangle+\frac{\partial P}{\partial \mathbf{q}}=0, \quad(t \geq 0),
$$

where $\mathbb{M}$ is the mass matrix and $\Gamma$ is a rank-3 tensor, usually called Christoffel symbol in Lagrangian Mechanics. If we denote by $M_{i j}$ the elements of the mass matrix (depending on $\mathbf{q}$ ) we have:

$$
\Gamma_{i j}^{k}:=\frac{1}{2}\left(\frac{M_{k i}}{\partial q_{j}}+\frac{M_{k j}}{\partial q_{i}}-\frac{M_{i j}}{\partial q_{k}}\right) .
$$

We next compute explicitly the elements $\Gamma_{i j}^{k}$ in terms of the potential of the fluid and the geometry of the solids, this task requiring formula of shape sensitivity analysis. The expression we obtain, well suited for numerical simulations, is the first novelty brought in in this paper.

In the next section, we consider a flow with non zero circulation. Circulation is handled as additional degrees of freedom of our system. We show that the ODEs governing the circulation can be decoupled from those driving the motion of the solids and explicitly solved. Thence, generalized coordinates related to the circulation can be canceled in the Euler-Lagrange equations that finally keep the same general form as (1.4):

$$
\mathbb{M} \ddot{\mathbf{q}}+\langle\Gamma(\mathbf{q}), \dot{\mathbf{q}}, \dot{\mathbf{q}}\rangle+\frac{\partial \widetilde{P}}{\partial \mathbf{q}}=0, \quad(t \geq 0),
$$

but where the potential energy contains an extra term du to the non zero fluid circulation. Its computation requires also the use of shape derivative formula. To our knowledge, such model involving circulation is new as well and is the second novelty of this paper.

We are next concerned with the well posedness of system of ODEs 1.5 . We prove that indeed it is, assuming that the boundaries of the solids are smooth enough. Further, we show that the solution is analytic and can be continued indefinitely unless a collision between two bodies or between a body with a fixed boundary of the fluid domain occurs. Such result has already be obtained in a more general case in 26, but the proof we give here has been significantly simplified.

Unlike to what happens in a viscous fluid, collisions are allowed with our model as proved in 11. How to handle collisions is the aim of the following section. It is done by adding to the Lagrangian an electrostatic-like potential energy whose electric charges would be located along the boundaries of the solids and generate a repulsive force. The potential energy must be set for the repulsive force to be neglectful when the solids are far apart but very strong when they go close in order to avoid collisions. Such refinement in the model is another novelty of this work.

Then we address the case of articulated bodies as described above by introducing holonomic constraints between the solids. We use the work already done 
for a set of solids to derive the expanded form of the Euler-Lagrange equation (1.3). The equation we eventually obtain is a generalization of the one given in 13. when hypotheses (H1-4) have been relaxed and buoyancy taken into account. We study the well posedness of this equation and get results roughly similar to the previous ones, related to rigid solids: existence of smooth solutions up to an hypothetic collision.

It is classical in the literature to use as controls directly the shape-changes of the bodies although a more realistic and interesting control would be the internal forces and torques causing these deformations. About this question, we first explain how to compute a posteriori the internal forces, the shape-changes being given. Then we prove that there is a one-to-one correspondence between the shape-changes and the internal forces they are du to. This new result entails the equivalence between choosing as controls the shape-changes or the internal forces what is of crucial importance for future works about the controllability of our model.

The two last sections of the article deal with numerical features of our works. In the first one we detail the numerical scheme a Matlab toolbox (Biohydrodynamics Toolbox) we have developed is based on. It consists in an ODE solver coupled with an integral equations solver for the computation of the fluid potential. Integral equations are numerically solved by applying the Nyström method, which is fast and accurate. An important point is that the elements of the mass matrices and the shape derivatives arising in the Euler-Lagrange equations can all of them be computed by knowing only the values of the fluid potential along the boundaries of the solids. It entails that there is no need to mesh the $2 \mathrm{~d}$ fluid domain but only its $1 \mathrm{~d}$ boundary.

In the last section we go further in the presentation of the Matlab toolbox. As an illustrative example we show that a result established by Sparenberg in [28, page 63] and ensuring that: a body of finite extent, moving periodically through an inviscid and incompressible fluid without shedding vorticity, cannot exert a force with non-zero mean value, is no longer true when the fluid contains fixed obstacles. This result is important because it proves that a fish is not only able to propel itself without shedding vorticity but can also generate a non-zero mean thrust what contradicts some common beliefs.

\section{Dynamics of a set of submerged rigid solids}

\section{$2.1 \quad$ Notation}

Let $\left(\mathbf{e}_{1}, \mathbf{e}_{2}\right)$ denote a reference Galilean frame by which we identify the physical space to $\mathbb{R}^{2}$. In this frame, the coordinates are denoted $\mathbf{x}:=\left(x_{1}, x_{2}\right)^{\top}$. At any time $t>0$, we denote by $\mathcal{S}^{i}(i \in\{1, \ldots, n\})$ the open domain occupied by the $i$-th solid. We set $\mathcal{S}:=\cup_{i} \mathcal{S}^{i}$ and $\mathcal{F}$ stands for the open region of the fluid. With this notation $\mathcal{M}:=\mathcal{F} \cup \overline{\mathcal{S}}$, as being the domain of the overall system fluid-solids, is independent of time. All of the domains are denoted at the initial time $t=0$ with subscript naught: $\mathcal{S}_{0}^{i}, \mathcal{S}_{0}$ and $\mathcal{F}_{0}$. We assume that 
$\partial \mathcal{F}_{0}$ is Lipschitz continuous and since $\mathcal{F}$ undergoes only rigid deformations, $\partial \mathcal{F}$ keeps the same regularity for all time. We assume also that the solids do not touch each other neither the fixed boundaries of the fluid at the initial time what means that $\overline{\mathcal{S}}_{0}^{i} \cap \overline{\mathcal{S}}_{0}^{j}=\emptyset$ if $i \neq j$ and $\overline{\mathcal{S}}_{0}^{i} \subset \mathcal{M}$ for all $i \in\{1, \ldots, n\}$. We denote by $\mathbf{n}=\left(n_{1}, n_{2}\right)^{\top}$ the unitary normal vector to $\partial \mathcal{F}$ directed toward the exterior of the fluid. The regularity of $\partial \mathcal{F}$ ensures that $\mathbf{n}$ is well defined almost everywhere. Likewise, we introduce the unitary tangent vector $\boldsymbol{\tau}=\left(\tau_{1}, \tau_{2}\right)^{\top}$ to $\partial \mathcal{F}$ and we choose its orientation such that $\{\mathbf{n}, \boldsymbol{\tau}\}$ be a direct basis. When $\partial \mathcal{F}$ is more regular, namely at least $C^{1,1}$ (continuously differentiable with first derivative Lipschitz continuous), we can also consider the curvature $\kappa$ defined almost everywhere by: $\partial_{\boldsymbol{\tau}} \mathbf{n}=-\kappa \boldsymbol{\tau}$.

Attached to each body, we define moving frames $\left(\mathbf{E}_{1}^{i}, \mathbf{E}_{2}^{i}\right)$. For any $i$, we choose it such that its origin coincides at any time with the center of mass of the solid and we denote with capital letters $\mathbf{X}^{i}:=\left(X_{1}^{i}, X_{2}^{i}\right)^{\top}$ the related body coordinates.

\subsection{Rigid motion}

Rigid displacements are described by elements $g$ of $G:=\mathrm{SE}(2)$ (the rigid body or euclidian group) consisting of pairs $(R, \mathbf{r})$ where $R \in \mathrm{SO}(2)$ is a rotation matrix and $\mathbf{r}:=\left(r_{1}, r_{2}\right)^{\top} \in \mathbb{R}^{2}$ is a vector. They would sometimes be identified with the matrix:

$$
g=\left(\begin{array}{cc}
R & \mathbf{r} \\
0 & 1
\end{array}\right),
$$

and for any vector $\mathbf{x}=\left(x_{1}, x_{2}\right)^{\top} \in \mathbb{R}^{2}$, we denote $g \mathbf{x}$ the vector such that:

$$
\left(\begin{array}{c}
g \mathbf{x} \\
1
\end{array}\right):=\left(\begin{array}{cc}
R & \mathbf{r} \\
0 & 1
\end{array}\right)\left(\begin{array}{c}
\mathbf{x} \\
1
\end{array}\right) .
$$

In the sequel, we will made no difference in the notation between the pair $(R, \mathbf{r})$ and the associated isometry of $\mathbb{R}^{2}$ defined by 2.1 . For all $(R, \dot{R}) \in \operatorname{TSO}(2)$ (the tangent bundle to $\mathrm{SO}(2)$ ), the matrix $\dot{R} R^{1}$ is skew-symmetric and hence, as it is classical in Solid Mechanics, there exists $\omega \in \mathbb{R}$ such that:

$$
\dot{R} R^{\top} \mathbf{x}=\omega \mathbf{x}^{\perp}, \quad \forall \mathbf{x} \in \mathbb{R}^{2},
$$

where $\mathbf{x}^{\perp}:=\left(-x_{2}, x_{1}\right)^{\top}$ stands the vector $\mathbf{x}:=\left(x_{1}, x_{2}\right)^{\top}$ positively quarter turned. The manifold $\mathrm{SO}(2)$ will be parameterized by means of matrices $R(\theta)$ $(\theta \in \mathbb{R} / 2 \pi)$ defined by:

$$
R(\theta):=\left(\begin{array}{cc}
\cos (\theta) & -\sin (\theta) \\
\sin (\theta) & \cos (\theta)
\end{array}\right)
$$

Thereby $\operatorname{TSO}(2)$ is parameterized by pairs $(\theta, \dot{\theta}) \in \mathbb{R} / 2 \pi \times \mathbb{R}$ and $\omega=\dot{\theta}$ in $(2.2)$. Likewise $\mathrm{G}$ is parameterized by $(\theta, \mathbf{r}) \in \mathbb{R} / 2 \pi \times \mathbb{R}^{2}$ and TG by $(\theta, \mathbf{r}, \omega, \dot{\mathbf{r}}) \in$ $\left(\mathbb{R} / 2 \pi \times \mathbb{R}^{2}\right) \times\left(\mathbb{R} \times \mathbb{R}^{2}\right)$. 
Going back to our problem, at any time there exists $g^{i}:=\left(R^{i}, \mathbf{r}^{i}\right) \in \operatorname{SE}(2)$ such that

$$
\mathbf{E}_{j}^{i}=R^{i} \mathbf{e}_{j},
$$

while $\mathbf{r}^{i}$ gives the location of the center of mass of the solid (see figure 1). With

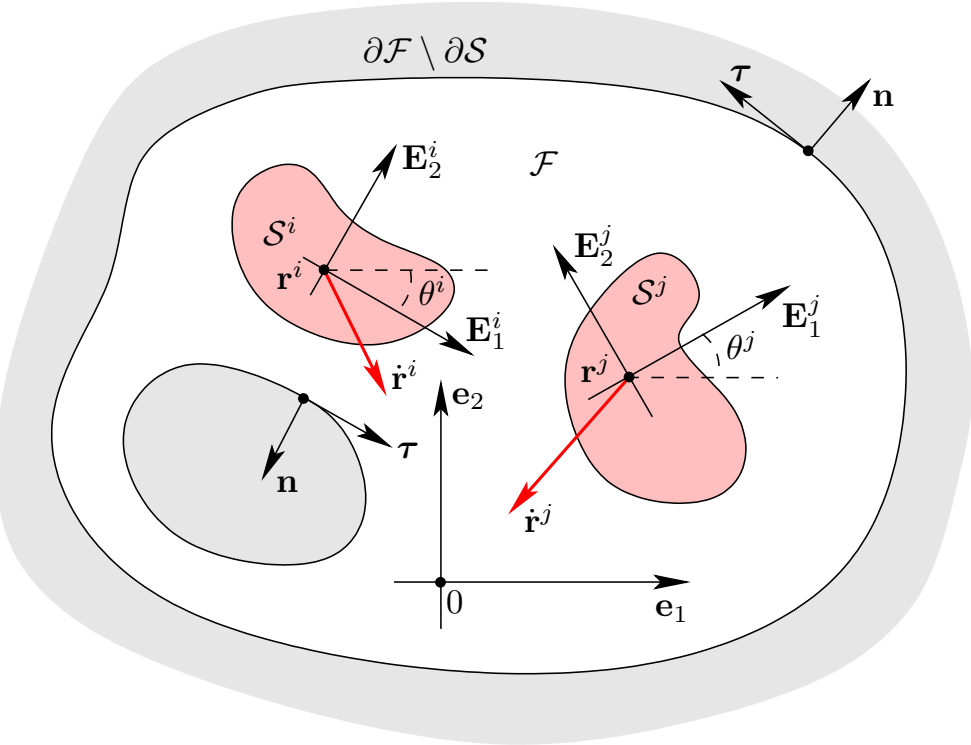

Figure 1: The fluid domain is denoted $\mathcal{F}$, the $i$-th solid occupies the domain $\mathcal{S}^{i}$, $\mathcal{S}=\cup_{i} \mathcal{S}^{i}$ and $\mathcal{M}=\mathcal{F} \cup \mathcal{S}$. The coordinates of the center of mass of the $i$-th solid in the reference frame $\left(\mathbf{e}_{k}\right)$ are $\mathbf{r}^{i}:=\left(r_{1}^{i}, r_{2}^{i}\right)^{\top}$. The frame $\left(\mathbf{E}_{k}^{i}\right)$ is attached to the solid and $\theta^{i}$ gives its orientation with respect to $\left(\mathbf{e}_{k}\right)$. At the time $t=0$, the domains and coordinates are denoted with subscript naught: $\mathcal{F}_{0}, \mathcal{S}_{0}^{i}, \mathbf{r}_{0}^{i}$ and so on.

our notation we have furthermore:

$$
\mathbf{x}=g^{i} \mathbf{X}^{i}
$$

and $\mathcal{S}^{i}=g^{i}\left(\mathcal{S}_{0}^{i}\right)$. We deduce also the relation between the area elements:

$$
\mathrm{d} \mathbf{x}=\mathrm{d} \mathbf{X}^{i} .
$$

Differentiating with respect to time identity (2.3), we deduce that the eulerian velocity of the $i$-th body is:

$$
\begin{aligned}
\mathbf{v}^{i}(\mathbf{x}): & =\dot{g}^{i} g^{i-1} \mathbf{x} \\
& =\omega^{i}\left(\mathbf{x}-\mathbf{r}^{i}\right)^{\perp}+\dot{\mathbf{r}}^{i}, \quad\left(\mathbf{x} \in \mathcal{S}^{i}\right) .
\end{aligned}
$$

We next use the partition $\cup_{i} \mathcal{S}^{i}$ of $\mathcal{S}$ to define in $\mathcal{S}$ the overall eulerian rigid velocity $\mathbf{v}$ of the solids by setting:

$$
\mathbf{v}(\mathbf{x})=\mathbf{v}^{i}(\mathbf{x}), \quad \mathbf{x} \in \mathcal{S}^{i} .
$$




\subsection{Fluid dynamics}

The flow being assumed to be irrotational, the eulerian velocity of the fluid can be written at any time $t$ as the gradient $\nabla \psi$ of a harmonic potential function $\psi$ defined in $\mathcal{F}$. The classical non-penetrating condition for inviscid fluids yields Neumann boundary conditions for the function $\psi$, namely:

$$
\begin{array}{ll}
\partial_{\mathbf{n}} \psi=\mathbf{v} \cdot \mathbf{n} & \text { on } \partial \mathcal{S}, \\
\partial_{\mathbf{n}} \psi=0 & \text { on } \partial \mathcal{F} \backslash \partial \mathcal{S} .
\end{array}
$$

For the Neumann boundary value problem to be well-posed, we must add conditions ensuring the uniqueness of the solution. We choose for instance:

$$
\int_{\partial \mathcal{S}} \psi \mathrm{d} \sigma=0
$$

Results about the well-posedness of such problem are given in the Appendix, Section A

For any solid $i$, we define three elementary potential functions: $\psi_{1}^{i}, \psi_{2}^{i}$ and $\psi_{3}^{i}$, all of them being harmonic in $\mathcal{F}$ and satisfying Neumann boundary conditions on $\partial \mathcal{F}$, namely, adopting the convention that $\partial_{\mathbf{n}} \psi_{j}^{i}=0$ on all of the unspecified boundaries:

$$
\begin{aligned}
& \partial_{\mathbf{n}} \psi_{1}^{i}=\mathbf{e}_{1} \cdot \mathbf{n} \quad \text { on } \partial \mathcal{S}^{i}, \\
& \partial_{\mathbf{n}} \psi_{2}^{i}=\mathbf{e}_{2} \cdot \mathbf{n} \quad \text { on } \partial \mathcal{S}^{i}, \\
& \partial_{\mathbf{n}} \psi_{3}^{i}=\left(\mathbf{x}-\mathbf{r}^{i}\right)^{\perp} \cdot \mathbf{n} \quad \text { on } \partial \mathcal{S}^{i} .
\end{aligned}
$$

We invoke then Kirchhoff's law to end up with the following decomposition of the overall potential:

$$
\psi:=\sum_{i} \dot{r}_{1}^{i} \psi_{1}^{i}+\dot{r}_{2}^{i} \psi_{2}^{i}+\omega^{i} \psi_{3}^{i}
$$

\subsection{Lagrangian function}

We denote by $\rho^{i}>0$ the density of the $i$-th solid while $\rho_{f}>0$ stands for the constant density of the fluid. The mass and the momentum of inertia of the $i$-th body are respectively:

$$
m^{i}:=\int_{\mathcal{S}^{i}} \rho^{i} \mathrm{~d} \mathbf{x}, \quad I^{i}:=\int_{\mathcal{S}^{i}} \rho^{i}\left|\mathbf{x}-\mathbf{r}^{i}\right|^{2} \mathrm{~d} \mathbf{x} .
$$

The kinematic energy of the $i$-th solid is:

$$
K^{i}:=\frac{1}{2} m^{i}\left|\mathbf{r}^{i}\right|^{2}+\frac{1}{2} I^{i}\left|\omega^{i}\right|^{2},
$$

and its potential energy du to its buoyancy reads:

$$
P^{i}:=g\left(m^{i} \mathbf{r}^{i}-m_{f}^{i} \mathbf{r}_{f}^{i}\right) \cdot \mathbf{e}_{2},
$$


where $g$ stands here for the standard gravity, $m_{f}^{i}>0$ is the mass of fluid occupying the same volume as the solid and $\mathbf{r}_{f}^{i}$ is its center of buoyancy defined by:

$$
m_{f}^{i}:=\rho_{f} \int_{\mathcal{S}^{i}} \mathrm{~d} \mathbf{x}, \quad \mathbf{r}_{f}^{i}:=\frac{\rho_{f}}{m_{f}^{i}} \int_{\mathcal{S}^{i}} \mathbf{x} \mathrm{d} \mathbf{x} .
$$

When $\rho^{i}$ is constant in $\mathcal{S}^{i}$ then $\mathbf{r}_{f}^{i}=\mathbf{r}^{i}$. On the other hand, the kinematic energy of the fluid is:

$$
K^{f}:=\frac{1}{2} \rho_{f} \int_{\mathcal{F}}|\nabla \psi|^{2} \mathrm{~d} \mathbf{x},
$$

and we deduce that the expression of the Lagrangian function of the sytem fluid-bodies:

$$
L:=K^{f}+\sum_{i}\left(K^{i}-P^{i}\right) .
$$

\subsection{Generalized coordinates and mass matrices}

Let us introduce the generalized coordinates:

$$
\mathbf{q}^{i}:=\left(r_{1}^{i}, r_{2}^{i}, \theta^{i}\right)^{\top} \quad \text { and } \quad \mathbf{q}:=\left(\mathbf{q}^{1}, \ldots, \mathbf{q}^{n}\right)^{\top},
$$

and likewise the generalized velocities $\dot{\mathbf{q}}^{i}$ and $\dot{\mathbf{q}}$. At the time $t=0$, they becomes:

$$
\mathbf{q}_{0}^{i}:=\left(\mathbf{r}_{0}^{i}, \theta_{0}^{i}\right)^{\top} \quad \text { and } \quad \mathbf{q}_{0}:=\left(\mathbf{q}_{0}^{1}, \ldots, \mathbf{q}_{0}^{n}\right)^{\top} .
$$

The components of $\mathbf{q}$ could be subsequently either denoted by $q_{i}$ with $i \in$ $\{1, \ldots, 3 n\}$ or $q_{j}^{i}$ with $i \in\{1, \ldots, n\}$ and $j \in\{1,2,3\}$ to emphasize the number of the solid we are referring to. We denote by $\mathcal{Q}$ the open subset of $\left(\mathbb{R}^{2} \times \mathbb{R} / 2 \pi\right)^{n}$ consisting of all the allowable $\mathbf{q}$ i.e. all the $\mathbf{q}$ for which the solids do not touch or overlap each other neither the boundary of the fluid domain. The set $\mathcal{Q}$ being an open set of a Banach space can also be seen as an analytic manifold and we denote $T \mathcal{Q}$ its tangent bundle consisting of the pairs $(\mathbf{q}, \dot{\mathbf{q}})$.

The generalized coordinates will allow us to rewrite the Lagrangian function in a convenient short form. Beforehand, we need to introduce the mass matrices of our system. The mass matrix of the $i$-th body is defined as being the diagonal matrix:

$$
\mathbb{M}_{r}^{i}:=\operatorname{diag}\left(m^{i}, m^{i}, I^{i}\right),
$$

while the added mass matrix du to the interaction between the $i$-th and the $j$-th solids in the fluid reads:

$$
\mathbb{M}_{i j}^{f}:=\rho_{f}\left[\begin{array}{ccc}
\int_{\mathcal{F}} \nabla \psi_{1}^{i} \cdot \nabla \psi_{1}^{j} \mathrm{~d} \mathbf{x} & \ldots & \int_{\mathcal{F}} \nabla \psi_{1}^{i} \cdot \nabla \psi_{3}^{j} \mathrm{~d} \mathbf{x} \\
\vdots & & \vdots \\
\int_{\mathcal{F}} \nabla \psi_{3}^{i} \cdot \nabla \psi_{1}^{j} \mathrm{~d} \mathbf{x} & \ldots & \int_{\mathcal{F}} \nabla \psi_{3}^{i} \cdot \nabla \psi_{3}^{j} \mathrm{~d} \mathbf{x}
\end{array}\right]
$$


The overall virtual mass matrix of the system fluid-solids is next defined as being the bloc matrix:

$$
\mathbb{M}:=\left[\begin{array}{cccc}
\mathbb{M}_{r}^{1}+\mathbb{M}_{11}^{f} & \mathbb{M}_{12}^{f} & \ldots & \mathbb{M}_{1 n}^{f} \\
\mathbb{M}_{21}^{f} & \mathbb{M}_{r}^{2}+\mathbb{M}_{22}^{f} & \ldots & \mathbb{M}_{2 n}^{f} \\
\vdots & \vdots & \ddots & \vdots \\
\mathbb{M}_{n 1}^{f} & \mathbb{M}_{n 2}^{f} & \ldots & \mathbb{M}_{r}^{n}+\mathbb{M}_{n n}^{f}
\end{array}\right]
$$

The overall kinetic energy of the system fluid-solids can now be rewritten in a very short form as a product matrix-vectors:

$$
K:=\frac{1}{2} \dot{\mathbf{q}}^{\top} \mathbb{M} \dot{\mathbf{q}}
$$

and if we set furthermore:

$$
P:=\sum_{i} P^{i}
$$

the expression of the Lagrangian function turns out to be:

$$
L:=K-P .
$$

Observe that in the definition 2.9 , the elements of the matrix $\mathbb{M}^{f}$ can be rewritten upon an integration by parts in the form:

$$
\int_{\mathcal{F}} \nabla \psi_{k}^{i} \cdot \nabla \psi_{l}^{j} \mathrm{~d} \mathbf{x}=\frac{1}{2} \int_{\partial \mathcal{S}^{j}} \psi_{k}^{i} \partial_{\mathbf{n}} \psi_{l}^{j} \mathrm{~d} \sigma+\frac{1}{2} \int_{\partial \mathcal{S}^{i}} \psi_{l}^{j} \partial_{\mathbf{n}} \psi_{k}^{i} \mathrm{~d} \sigma
$$

involving only integrals on the boundaries of the solids.

\subsection{Euler-Lagrange equation}

The Lagrangian is a function of $\mathbf{q}$ and $\dot{\mathbf{q}}$ only. We invoke now Hamilton's principle which tells us that the Euler-Lagrange equation driving the dynamics of our system is:

$$
\frac{d}{d t} \frac{\partial L}{\partial \dot{\mathbf{q}}}-\frac{\partial L}{\partial \mathbf{q}}=0, \quad(t \geq 0)
$$

Expanding this equality, taking into account the expressions 2.10, we get a second order non-linear ODE:

$$
\mathbb{M} \ddot{\mathbf{q}}+\langle\Gamma(\mathbf{q}), \dot{\mathbf{q}}, \dot{\mathbf{q}}\rangle+\frac{\partial P}{\partial \mathbf{q}}=0, \quad(t \geq 0),
$$

in which $\Gamma(\mathbf{q})$ is a rank-3 tensor usually called Christoffel symbol in Lagrangian Mechanics. Denoting by $M_{i j}$ the elements of the virtual mass matrix $\mathbb{M}(i, j \in$ $\{1, \ldots, 3 n\})$ and by $q_{i}$ the elements of $\mathbf{q}(i \in\{1, \ldots, 3 n\})$, it is defined by:

$$
\Gamma_{i j}^{k}:=\frac{1}{2}\left(\frac{\partial M_{k i}}{\partial q_{j}}+\frac{\partial M_{k j}}{\partial q_{i}}-\frac{\partial M_{i j}}{\partial q_{k}}\right), \quad(i, j, k \in\{1, \ldots, 3 n\}) .
$$


Considering the expression of the matrix $\mathbb{M}$, we realize that the main difficulty lies in computing the derivatives with respect to the generalized coordinates of the elements $M_{i j}^{f}$ of the added mass matrix $\left(i=3\left(i^{\prime}-1\right)^{\prime}+i^{\prime \prime}, j=3\left(j^{\prime}-1\right)+j^{\prime \prime}\right.$, $\left.i^{\prime}, j^{\prime} \in\{1, \ldots, n\}, i^{\prime \prime}, j^{\prime \prime} \in\{1,2,3\}\right)$ :

$$
M_{i j}^{f}:=\rho_{f} \int_{\mathcal{F}} \nabla \psi_{i^{\prime \prime}}^{i^{\prime}} \cdot \nabla \psi_{j^{\prime \prime}}^{j^{\prime}} \mathrm{d} \mathbf{x},
$$

since such quantities depend on q not only because the domain of integration $\mathcal{F}$ does but also because the elementary potentials are defined as solutions of boundary problems in $\mathcal{F}$. Although quite involved, formulae to compute such derivatives are available and to display them we need to introduce some additional notations.

We use in the sequel results and notation given in the Appendix, Section B We denote by $\mathbf{w}_{k}^{i}(i \in\{1, \ldots, n\}$ and $k \in\{1,2,3\})$ the velocity fields defined on $\partial \mathcal{S}^{i}$ by:

$$
\mathbf{w}_{1}^{i}:=\mathbf{e}_{1}, \quad \mathbf{w}_{2}^{i}:=\mathbf{e}_{2}, \quad \mathbf{w}_{3}^{i}:=\left(\mathbf{x}-\mathbf{r}^{i}\right)^{\perp},
$$

so that boundary conditions (2.6) can be rewritten $\partial_{\mathbf{n}} \psi_{k}^{i}=\mathbf{w}_{k}^{i} \cdot \mathbf{n}$ for all $i$ and $k$. Let us introduce also for all $i \in\{1, \ldots, n\}$ the functions $\boldsymbol{\xi}^{i}, \boldsymbol{\zeta}^{i}: \partial \mathcal{S}^{i} \rightarrow \mathbb{R}^{3}$, defined by:

$$
\boldsymbol{\xi}^{i}:=\left(\begin{array}{c}
-n_{2} \\
n_{1} \\
\left(\mathbf{x}-\mathbf{r}^{i}\right) \cdot \mathbf{n}
\end{array}\right), \quad \boldsymbol{\zeta}^{i}:=\left(\begin{array}{c}
n_{1} \\
n_{2} \\
\left(\mathbf{x}-\mathbf{r}^{i}\right)^{\perp} \cdot \mathbf{n}
\end{array}\right),
$$

and the matrices defined on $\partial \mathcal{S}^{i}$ as well by:

$$
\mathbb{S}^{i}:=\kappa\left(\boldsymbol{\xi}^{i} \otimes \boldsymbol{\xi}^{i}-\boldsymbol{\zeta}^{i} \otimes \boldsymbol{\zeta}^{i}\right)+\boldsymbol{\xi}^{i} \otimes \mathbf{f}_{3}+\mathbf{f}_{3} \otimes \boldsymbol{\xi}^{i}-\left[\left(\mathbf{x}-\mathbf{r}^{i}\right) \cdot \mathbf{n}\right]\left(\mathbf{f}_{3} \otimes \mathbf{f}_{3}\right),
$$

where $\mathbf{f}_{3}:=(0,0,1)^{\top}$. At last, we recall the definition of the Kronecker symbol:

$$
\delta_{j}^{i}:= \begin{cases}1 & \text { if } i=j, \\ 0 & \text { otherwise }\end{cases}
$$

and we claim that:

Proposition 2.1 The quantities (2.14) are analytic with respect to $\mathbf{q}$. If $\partial \mathcal{F}$ is of class $C^{1,1}$, the Christoffel symbols $(2.13)$ can be computed in terms of the elementary potentials. Thus, we get for $k=3\left(k^{\prime}-1\right)+k^{\prime \prime}, i=3\left(i^{\prime}-1\right)+i^{\prime \prime}$ and $j=3\left(j^{\prime}-1\right)+j^{\prime \prime}$ with $k^{\prime}, i^{\prime}, j^{\prime} \in\{1, \ldots, n\}$ and $k^{\prime \prime}, i^{\prime \prime}, j^{\prime \prime} \in\{1,2,3\}$ (what means that $k^{\prime}, i^{\prime}$ and $j^{\prime}$ are the indices of the solids):

$$
\begin{aligned}
\Gamma_{i j}^{k}=- & \frac{\rho_{f}}{2} \int_{\partial \mathcal{S}^{i^{\prime}}} \partial_{\boldsymbol{\tau}} \psi_{k^{\prime \prime}}^{k^{\prime}} \partial_{\boldsymbol{\tau}} \psi_{j^{\prime \prime}}^{j^{\prime}}\left(\mathbf{w}_{i^{\prime \prime}}^{i^{\prime}} \cdot \mathbf{n}\right) \mathrm{d} \sigma-\frac{\rho_{f}}{2} \int_{\partial \mathcal{S}^{\prime}} \partial_{\boldsymbol{\tau}} \psi_{k^{\prime \prime}}^{k^{\prime}} \partial_{\boldsymbol{\tau}} \psi_{i^{\prime \prime}}^{i^{\prime}}\left(\mathbf{w}_{j^{\prime \prime}}^{j^{\prime}} \cdot \mathbf{n}\right) \mathrm{d} \sigma \\
+ & \frac{\rho_{f}}{2} \int_{\partial \mathcal{S}^{k^{\prime}}} \partial_{\boldsymbol{\tau}} \psi_{i^{\prime \prime}}^{i^{\prime}} \partial_{\boldsymbol{\tau}} \psi_{j^{\prime \prime}}^{j^{\prime}}\left(\mathbf{w}_{k^{\prime \prime}}^{k^{\prime}} \cdot \mathbf{n}\right) \mathrm{d} \sigma+\delta_{i^{\prime}}^{j^{\prime}} \rho_{f} \int_{\partial \mathcal{S}^{i^{\prime}}} \psi_{k^{\prime \prime}}^{k^{\prime}} \mathbb{S}_{i^{\prime \prime} j^{\prime \prime}}^{i^{\prime}} \mathrm{d} \sigma \\
+\delta_{i^{\prime}}^{j^{\prime}} & \delta_{i^{\prime}}^{k^{\prime}} \frac{\rho_{f}}{2} \int_{\partial \mathcal{S}^{\prime}}\left(\mathbf{w}_{k^{\prime \prime}}^{k^{\prime}} \cdot \mathbf{n}\right)\left(\mathbf{w}_{i^{\prime \prime}}^{i^{\prime}} \cdot \mathbf{n}\right)\left(\mathbf{w}_{j^{\prime \prime}}^{j^{\prime}} \cdot \mathbf{n}\right) \mathrm{d} \sigma
\end{aligned}
$$


Notice that, like for the elements of the mass matrix, all of the terms in 2.15 are integrals on the boundaries of the solids.

Proof: We study the sensitivity of the elements (2.14) of the matrix $\mathbb{M}^{f}$ at the time $t=0$, the process being similar at any time. We wish to apply results of Section B so we need to define the mapping $\phi_{s}$ arising therein.

Since the solids do no touch each other at the time $t=0$, we can consider open domains $\mathcal{S}_{\varepsilon}^{i}$ and $\mathcal{O}^{i}$ such that $\overline{\mathcal{S}}_{0}^{i} \subset \mathcal{S}_{\varepsilon}^{i}, \overline{\mathcal{S}}_{\varepsilon}^{i} \subset \mathcal{O}^{i}, \overline{\mathcal{O}}^{i} \subset \mathcal{M}$ for all $i \in$ $\{1, \ldots, n\}$ and $\overline{\mathcal{O}}^{i} \cap \overline{\mathcal{O}}^{j}=\emptyset$ for all $i \neq j$. Invoking Urisohn's Lemma, there exists a function $\chi$ of class $C^{\infty}$, compactly supported and such that $0 \leq \chi \leq 1$, $\chi=1$ in $\mathcal{S}_{\varepsilon}^{i}$ and $\chi=0$ in $\mathbb{R}^{2} \backslash \cup_{i} \overline{\mathcal{O}}^{i}$. With the notation of Section $\mathrm{B}$, we specialize $s=\mathbf{q}$ and we set:

$$
\phi_{s}(\mathbf{x}):= \begin{cases}(1-\chi(\mathbf{x})) \mathbf{x}+\chi(\mathbf{x})\left(R\left(\theta^{i}\right)\left(\mathbf{x}-\mathbf{r}_{0}^{i}\right)+\mathbf{r}^{i}\right) & \text { if } \mathbf{x} \in \overline{\mathcal{O}}^{i}, \\ \mathbf{x} & \text { if } \mathbf{x} \in \mathbb{R}^{2} \backslash \cup_{i} \overline{\mathcal{O}}^{i} .\end{cases}
$$

For all $\mathbf{q}$ close enough to $\mathbf{q}_{0}$, the mapping $\phi_{s}$ is an infinitely differentiable diffeomorphism from $\mathbb{R}^{2}$ onto itself and

$$
\phi_{s}(\mathbf{x})=R\left(\theta^{i}\right)\left(\mathbf{x}-\mathbf{r}_{0}^{i}\right)+\mathbf{r}^{i} \quad \text { in } \mathcal{S}_{\varepsilon}^{i} .
$$

We next apply Proposition B.1 to get the analyticity of quantities 2.14).

We wish then to apply Proposition B.3. To this purpose, we need to compute the quantities involved in formula (B.3). Straightforward computations yield, on $\partial \mathcal{S}^{i}$ and for all $i \in\{1, \ldots, n\}$ :

$$
\begin{array}{ll}
\mathbf{w}_{1}^{i} \cdot \boldsymbol{\tau}=-n_{2}, & \partial_{\boldsymbol{\tau}}\left(\mathbf{w}_{1}^{i} \cdot \mathbf{n}\right) \cdot \boldsymbol{\tau}=\kappa n_{2}, \\
\mathbf{w}_{2}^{i} \cdot \boldsymbol{\tau}=n_{1}, & \partial_{\boldsymbol{\tau}}\left(\mathbf{w}_{2}^{i} \cdot \mathbf{n}\right) \cdot \boldsymbol{\tau}=-\kappa n_{1}, \\
\mathbf{w}_{3}^{i} \cdot \boldsymbol{\tau}=\left(\mathbf{x}-\mathbf{r}^{i}\right) \cdot \mathbf{n}, & \partial_{\boldsymbol{\tau}}\left(\mathbf{w}_{3}^{i} \cdot \mathbf{n}\right) \cdot \boldsymbol{\tau}=(-1-\kappa)\left(\mathbf{x}-\mathbf{r}^{i}\right) \cdot \mathbf{n} .
\end{array}
$$

At last, we get:

$$
\gamma_{i j}(\mathbf{x})= \begin{cases}-\left(\mathbf{x}-\mathbf{r}^{i}\right) \cdot \mathbf{n} & \text { if } i=j=3 k, k \in\{1, \ldots, n\} \text { and } \mathbf{x} \in \mathcal{S}^{k}, \\ \mathbf{0} & \text { otherwise }\end{cases}
$$

and formula B.3 matches formula 2.15.

In 2.12, it remains only to compute the derivatives of the potential energy (2.8) with respect to the generalized coordinates. Straightforward computations yield:

$$
\frac{\partial P^{i}}{\partial q_{j}^{k}}= \begin{cases}g\left(m^{i}-m_{f}^{i}\right) & \text { if } i=k \text { and } j=2, \\ g\left(m^{i} \mathbf{r}^{i}-m_{f}^{i} \mathbf{r}_{f}^{i}\right)^{\perp} \cdot \mathbf{e}_{2} & \text { if } i=k \text { and } j=3, \\ 0 & \text { otherwise. }\end{cases}
$$




\section{Model with circulation}

Since there are $n$ submerged bodies, adding circulation to our model means adding $n$ degrees of freedom for the fluid flow. Let us introduce $n$ elementary stream functions $\varphi^{i}, i \in\{1, \ldots, n\}$, harmonic in $\mathcal{F}$ and satisfying Dirichlet boundary conditions:

$$
\begin{array}{ll}
\varphi^{i}=1 & \text { on } \partial \mathcal{S}^{i}, \\
\varphi^{i}=0 & \text { on } \partial \mathcal{F} \backslash \partial \mathcal{S}^{i} .
\end{array}
$$

At any time, the overall stream function related to the circulation can be written as a linear combination of the functions $\varphi^{i}$ :

$$
\varphi=\sum_{i} c^{i} \varphi^{i}
$$

where the coefficients $c^{i}$ are real for all $i \in\{1, \ldots, n\}$ and depend on time. We call $\mathbf{c}:=\left(c^{1}, \ldots, c^{n}\right)^{\top}$ the generalized circulation variable, homogeneous to a generalized velocity. The unknowns of our problem are now $\mathbf{q}$, $\dot{\mathbf{q}}$ and $\mathbf{c}$. The eulerian velocity of the fluid becomes when circulation is taken into account:

$$
\mathbf{u}:=\nabla \psi+\nabla \varphi^{\perp} \quad \text { in } \mathcal{F} .
$$

We defined the additional mass matrix:

$$
\mathbb{C}:=\rho_{f}\left[\begin{array}{ccc}
\int_{\mathcal{F}} \nabla \varphi^{1} \cdot \nabla \varphi^{1} \mathrm{~d} \mathbf{x} & \ldots & \int_{\mathcal{F}} \nabla \varphi^{1} \cdot \nabla \varphi^{n} \mathrm{~d} \mathbf{x} \\
\vdots & & \vdots \\
\int_{\mathcal{F}} \nabla \varphi^{n} \cdot \nabla \varphi^{1} \mathrm{~d} \mathbf{x} & \ldots & \int_{\mathcal{F}} \nabla \varphi^{n} \cdot \nabla \varphi^{n} \mathrm{~d} \mathbf{x}
\end{array}\right],
$$

and we point out that its elements can be rewritten, using Green's formula, in the form:

$$
\int_{\mathcal{F}} \nabla \varphi^{i} \cdot \nabla \varphi^{j} \mathrm{~d} \mathbf{x}=\frac{1}{2} \int_{\partial \mathcal{S}^{i}} \partial_{\mathbf{n}} \varphi^{j} \mathrm{~d} \sigma+\frac{1}{2} \int_{\partial \mathcal{S}^{j}} \partial_{\mathbf{n}} \varphi^{i} \mathrm{~d} \sigma .
$$

The virtual mass matrix of the system fluid-solids is now the bloc diagonal matrix:

$$
\left[\begin{array}{cc}
\mathbb{M} & 0 \\
0 & \mathbb{C}
\end{array}\right]
$$

because all of the terms involving both an elementary potential and an elementary stream function are null. Indeed, for any $i, k \in\{1, \ldots, n\}$ and $j \in\{1,2,3\}$ :

$$
\begin{aligned}
\int_{\mathcal{F}} \nabla \psi_{j}^{i} \cdot\left(\nabla \varphi^{k}\right)^{\perp} \mathrm{d} \mathbf{x} & =-\int_{\mathcal{F}}\left(\nabla \psi_{j}^{i}\right)^{\perp} \cdot \nabla \varphi^{k} \mathrm{~d} \mathbf{x} \\
& =-\int_{\partial \mathcal{S}^{k}}\left(\nabla \psi_{j}^{i}\right)^{\perp} \cdot \mathbf{n} \mathrm{d} \sigma \\
& =\int_{\partial \mathcal{S}^{k}} \partial_{\boldsymbol{\tau}} \psi_{j}^{i} \mathrm{~d} \sigma \\
& =0 .
\end{aligned}
$$


Observe that the elementary stream functions depend on the position of the solids and hence on q. We draw the same conclusion for the mass matrix $\mathbb{C}$. The Lagrangian function given in 2.10 rewrites now:

$$
L:=\frac{1}{2} \dot{\mathbf{q}}^{\top} \mathbb{M} \dot{\mathbf{q}}+\frac{1}{2} \mathbf{c}^{\top} \mathbb{C} \mathbf{c}-P .
$$

Once more, Hamilton's principle tells us that (remind that $\mathbf{c}$ is homogeneous to a generalized velocity):

which simplifies into:

$$
\frac{d}{d t} \frac{\partial L}{\partial \mathbf{c}}=0, \quad(t \geq 0)
$$

$$
\frac{d}{d t}(\mathbb{C} \mathbf{c})=0, \quad(t \geq 0)
$$

It means that the impulse of the fluid (see [17] for a definition) corresponding to the circulation is constant. We recover a well known result: if there is no circulation at the time $t=0$, the circulation remains null for all time. We denote $\Pi_{c}^{f} \in \mathbb{R}^{n}$ the initial impulse related to the circulation and we get:

$$
\mathbf{c}=\mathbb{C}^{-1} \Pi_{c}^{f}, \quad(t \geq 0) .
$$

As being a mass matrix, $\mathbb{C}$ is positive definite and hence always invertible. We introduce the modified potential energy:

$$
\widetilde{P}:=P+P_{c},
$$

with

$$
P_{c}:=-\frac{1}{2}\left(\Pi_{c}^{f}\right)^{\top} \mathbb{C}^{-1} \Pi_{c}^{f} .
$$

The Lagrangian function turns out to be:

$$
L:=\frac{1}{2} \dot{\mathbf{q}}^{\top} \mathbb{M} \dot{\mathbf{q}}-\widetilde{P}
$$

and the Euler-Lagrange equation 2.12 must be modified as follows:

$$
\mathbb{M} \ddot{\mathbf{q}}+\langle\Gamma(\mathbf{q}), \dot{\mathbf{q}}, \dot{\mathbf{q}}\rangle+\frac{\partial \widetilde{P}}{\partial \mathbf{q}}=0, \quad(t \geq 0)
$$

For all $k^{\prime} \in\{1, \ldots, n\}$ and $k^{\prime \prime} \in\{1,2,3\}$, we have:

$$
\frac{\partial P_{c}}{\partial q_{k^{\prime \prime}}^{k^{\prime}}}:=-\frac{1}{2}\left(\Pi_{c}^{f}\right)^{\top}\left(\mathbb{C}^{-1}\right)^{\top} \frac{\partial \mathbb{C}}{\partial q_{k^{\prime \prime}}^{k^{\prime}}} \mathbb{C}^{-1} \Pi_{c}^{f}
$$

The following Lemma allows to go further in the computation of this term:

Lemma 3.1 If $\partial \mathcal{F}$ is of class $C^{1,1}$, the elements of the matrix $\partial \mathbb{C} / \partial q_{k^{\prime \prime}}^{k^{\prime}}$ read:

$$
\frac{\partial}{\partial q_{k^{\prime \prime}}^{k^{\prime}}}\left(\int_{\mathcal{F}} \nabla \varphi^{i} \cdot \nabla \varphi^{j} \mathrm{~d} \mathbf{x}\right)=-\int_{\partial \mathcal{S}^{k^{\prime}}} \partial_{\mathbf{n}} \varphi^{i} \partial_{\mathbf{n}} \varphi^{j}\left(\mathbf{w}_{k^{\prime \prime}}^{k^{\prime}} \cdot \mathbf{n}\right) \mathrm{d} \sigma,
$$

for all $i, j \in\{1, \ldots, n\}$. 
Proof: We just give an outline of the proof, most of the arguments being similar to those of Section B Using the same notation as in the proof of Proposition 2.1 we first compute $\partial_{s_{i}} \varphi^{j}$ for all $i \in\{1, \ldots, 3 n\}$ and $j \in\{1, \ldots, n\}$. Straightforward computations yield:

$$
\begin{aligned}
-\Delta \partial_{s_{i}} \varphi^{j} & =0 & & \text { in } \mathcal{F}, \\
\partial_{s_{i}} \varphi^{j} & =0 & & \text { on } \partial \mathcal{F} \backslash \partial \mathcal{S}^{i^{\prime}},
\end{aligned}
$$

where $i=3\left(i^{\prime}-1\right)+i^{\prime \prime}, i^{\prime} \in\{1, \ldots, n\}, i^{\prime \prime} \in\{1,2,3\}$. Differentiating with respect to $s_{i}$ the remaining boundary condition:

$$
\varphi^{j}\left(\phi_{s}\right)=\delta_{j}^{i^{\prime}} \quad \text { on } \partial \mathcal{S}_{0}^{i^{\prime}},
$$

we get:

$$
\partial_{s_{i}} \varphi^{j}+\nabla \varphi^{j} \cdot \mathbf{w}_{i^{\prime \prime}}^{i^{\prime}}=0 \quad \text { on } \partial \mathcal{S}^{i^{\prime}} .
$$

But since $\varphi^{j}$ is constant along $\partial \mathcal{S}^{i^{\prime}}$, we have $\nabla \varphi^{j}=\left(\partial_{\mathbf{n}} \varphi^{j}\right) \mathbf{n}$ and the identity above turns into:

$$
\partial_{s_{i}} \varphi^{j}=-\partial_{\mathbf{n}} \varphi^{j}\left(\mathbf{w}_{i^{\prime \prime}}^{i^{\prime}} \cdot \mathbf{n}\right) \quad \text { on } \partial \mathcal{S}^{i^{\prime}} .
$$

Then, Reynolds's formula (see [10, Corollary 5.2.2 page 172]) yields for all $i \in$ $\{1, \ldots, 3 n\}$ and $j, k \in\{1, \ldots, n\}$ :

$$
\begin{aligned}
\frac{\partial}{\partial s_{i}}\left(\int_{\mathcal{F}} \nabla \varphi^{j} \cdot \nabla \varphi^{k} \mathrm{~d} \mathbf{x}\right) & =\int_{\partial \mathcal{S}^{i^{\prime}}}\left(\nabla \varphi^{j} \cdot \nabla \varphi^{k}\right)\left(\mathbf{w}_{i^{\prime \prime}}^{i^{\prime}} \cdot \mathbf{n}\right) \mathrm{d} \sigma \\
& +\int_{\mathcal{F}} \nabla\left(\partial_{s_{i}} \varphi^{j}\right) \cdot \nabla \varphi^{k} \mathrm{~d} \mathbf{x}+\int_{\mathcal{F}} \nabla \varphi^{j} \cdot \nabla\left(\partial_{s_{i}} \varphi^{k}\right) \mathrm{d} \mathbf{x} .
\end{aligned}
$$

Once more, we observe that $\nabla \varphi^{j}=\partial_{\mathbf{n}} \varphi^{j}$ and we apply Green's formula in the two latest terms to obtain formula (3.5).

From equations $(3.2)$ and $(3.3)$ we deduce that the motion of the solids in the fluid cannot generate circulation, if circulation is zero at the initial time, but the converse is false: starting from rest, solids immersed in a fluid with non zero circulation are certainly set into motion by the fluid.

\section{Well-posedness of the Euler-Lagrange equa- tions}

We study now the well-posedness of equations 2.12 and $(3.3)$. We have:

Theorem 4.1 For any initial data $\left(\mathbf{q}_{0}, \dot{\mathbf{q}}_{0}\right) \in T \mathcal{Q}$, there exists a unique solution $t \in[0, T) \mapsto \mathbf{q} \in \mathcal{Q}$ to equation (3.3) such that $\left(\mathbf{q}(0), \frac{d \mathbf{q}}{d t}(0)\right)=\left(\mathbf{q}_{0}, \dot{\mathbf{q}}_{0}\right)$. Further, the solution is analytic and either $T=+\infty$ or $T$ corresponds to the time of a collision between two solids or between a solid with any boundary of the fluid domain. 
Proof: Since $\mathcal{F}$ is at least Lipschitz-continuous then we claim that for all $i, j \in\{1, \ldots, n\}$ and $k, l \in\{1,2,3\}$, the quantities:

$$
\int_{\mathcal{F}} \nabla \psi_{k}^{i} \cdot \nabla \psi_{l}^{j} \mathrm{~d} \mathbf{x} \text { and } \int_{\mathcal{F}} \nabla \varphi^{i} \cdot \nabla \varphi^{j} \mathrm{~d} \mathbf{x}
$$

are analytic with respect to $\mathbf{q}$. It is a non-trivial result of shape sensitivity analysis which has already been proved in [26. It entails that all of the terms in (3.3) are analytic with respect to $\mathbf{q}$ and also with respect to $\dot{\mathbf{q}}$ for they are linear or quadratic with respect to this second variable.

The next argument is that the matrix $\mathbb{M}$ is definite-positive and hence invertible for all $\mathbf{q} \in \mathcal{Q}$. The Cauchy-Lipschitz Theorem applies to EDO (3.3) and ensures that for any initial data, there exists a unique analytic solution local in time.

It remains to prove that the solution $(\mathbf{q}, \dot{\mathbf{q}})$ cannot blow up in finite time unless a collision occurs. To this purpose, we introduce the overall energy of the system fluid-solids:

$$
E:=\frac{1}{2} \dot{\mathbf{q}}^{\top} \mathbf{M} \dot{\mathbf{q}}+\widetilde{P}
$$

Differentiating with respect to time the identity:

$$
E=\frac{\partial L}{\partial \dot{\mathbf{q}}} \cdot \dot{\mathbf{q}}-L,
$$

and applying the chain rule, we end up with the relation:

$$
\frac{d}{d t} E=\left(\frac{d}{d t} \frac{\partial L}{\partial \dot{\mathbf{q}}}-\frac{\partial L}{\partial \mathbf{q}}\right) \cdot \dot{\mathbf{q}}
$$

which means that $E$ is conserved. The solution $(\mathbf{q}, \dot{\mathbf{q}})$ is therefore bounded in any compact subset of $T \mathcal{Q}$. Classical results for ODEs tell that the solution can be continued up to a maximum time $T$ and that either $T=+\infty$ or:

$$
\lim _{t \rightarrow T}(\mathbf{q}, \dot{\mathbf{q}}) \in \partial(T \mathcal{Q})
$$

what means, since the tangent spaces are boundary-less vector spaces:

$$
\lim _{t \rightarrow T} \mathbf{q} \in \partial \mathcal{Q} .
$$

This last condition characterizes a collision (or a contact) and the proof is completed.

\section{$5 \quad$ Handling collisions}

Collisions between immersed rigid solids evolving freely in a potential flow may occur as proved in [11. In the numerical simulations, collisions and overlapping between solids will cause the computations to fail and hence have to be 
prevented. To this purpose, we add to our model an electrostatic-like potential energy defined by:

$$
V:=\varepsilon \sum_{i=0}^{n} \sum_{j=i+1}^{n} \int_{\partial \mathcal{S}^{j}} \int_{\partial \mathcal{S}^{i}}\left|\mathbf{x}-\mathbf{x}^{\prime}\right|^{-\alpha} \mathrm{d} \sigma_{\mathbf{x}} \mathrm{d} \sigma_{\mathbf{x}^{\prime}},
$$

where we have denoted $\partial \mathcal{S}^{0}:=\partial \mathcal{F} \backslash \partial \mathcal{S}$ (i.e. $\partial \mathcal{S}^{0}$ is the boundary of the fluid not shared with the solids) and $\varepsilon>0, \alpha>0$ are two constants. We choose them such that the repulsive force induced by the potential $V$ be neglectful when the solids are not very close. The potential $V$ must be subtracted to the right hand side term in the definition of the Lagrangian function and the Euler-Lagrange equation 3.3 becomes:

$$
\mathbb{M} \ddot{\mathbf{q}}+\langle\Gamma(\mathbf{q}), \dot{\mathbf{q}}, \dot{\mathbf{q}}\rangle+\frac{\partial \widetilde{P}}{\partial \mathbf{q}}+\frac{\partial V}{\partial \mathbf{q}}=0, \quad(t \geq 0) .
$$

We easily obtain that, for $k=3\left(k^{\prime}-1\right)+k^{\prime \prime},\left(k^{\prime} \in\{1, \ldots, n\}, k^{\prime \prime} \in\{1,2,3\}\right)$ :

$$
\frac{\partial V}{\partial q_{k^{\prime \prime}}^{k^{\prime}}}=2 \varepsilon \sum_{j \neq k^{\prime}} \int_{\partial \mathcal{S}^{j}} \int_{\partial \mathcal{S}^{k^{\prime}}}\left|\mathbf{x}-\mathbf{x}^{\prime}\right|^{-\alpha}\left[\frac{\left(\mathbf{x}-\mathbf{x}^{\prime}\right)}{\left|\mathbf{x}-\mathbf{x}^{\prime}\right|^{2}} \cdot \mathbf{n}+\kappa\right]\left(\mathbf{w}_{k^{\prime \prime}}^{k^{\prime}} \cdot \mathbf{n}\right) \mathrm{d} \sigma_{\mathbf{x}} \mathrm{d} \sigma_{\mathbf{x}^{\prime}}
$$

Once again, observe that this expression involves only integrals on the boundaries of the solids.

\section{Dynamics of articulated bodies}

To simplify, let us assume in this section that there is no circulation. The dynamics of articulated bodies can easily be deduced from the dynamics of a set a solids. Indeed, articulated bodies are defined as a collection of rigid solids linked together through holonomic constraints which are easily handled within Lagangian formalism. We write that:

$$
\mathbf{q}=F(\mathbf{p}, \mathbf{u}),
$$

where $\mathbf{p}:=\left(p_{1}, \ldots, p_{m}\right)$ belongs to $\mathcal{P}$ an analytic manifold of dimension $m$ $(1 \leq m<3 n)$ and stands for the new degrees of freedom of the system and $\mathbf{u}:=\left(u_{1}, \ldots, u_{d}\right) \in \mathcal{U}$ (an analytic manifold of dimension $d \geq 1$ such that $m+d=3 n)$ is a given control variable governing the relative position of the solids composing the articulated bodies. A simple example of such function is obtained by choosing for $\mathbf{p}$ some components of $\mathbf{q}$, the remaining ones being driven by a relation involving $\mathbf{p}$ and the control $\mathbf{u}$. To simplify we assume that $F$ is also analytic from $\mathcal{P} \times \mathcal{U}$ into $\mathcal{Q}$.

Remind that the Lagrangian $L=L(\mathbf{q}, \dot{\mathbf{q}})$ is a function of $\mathbf{q}$ and $\dot{\mathbf{q}}$ only. We define

$$
\mathcal{L}(\mathbf{p}, \mathbf{u}, \dot{\mathbf{p}}, \dot{\mathbf{u}}):=L\left(F(\mathbf{p}, \mathbf{u}), \frac{\partial F}{\partial \mathbf{p}} \dot{\mathbf{p}}+\frac{\partial F}{\partial \mathbf{u}} \dot{\mathbf{u}}\right)
$$


and the equation governing the motion of the articulated bodies is:

$$
\frac{d}{d t} \frac{\partial \mathcal{L}}{\partial \dot{\mathbf{p}}}-\frac{\partial \mathcal{L}}{\partial \mathbf{p}}=0, \quad(t \geq 0)
$$

Introducing the matrices:

$$
\mathbb{N}_{\mathbf{p}}:=\frac{\partial F}{\partial \mathbf{p}}, \quad \mathbb{N}_{\mathbf{u}}:=\frac{\partial F}{\partial \mathbf{u}}
$$

and the rank-3 tensor (the second derivative of $F$ ):

$$
\left\langle\mathbf{D}^{2} F, \dot{\mathbf{p}}, \dot{\mathbf{u}}\right\rangle:=\left\langle\frac{\partial^{2} F}{\partial \mathbf{p}^{2}}, \dot{\mathbf{p}}, \dot{\mathbf{p}}\right\rangle+\left\langle\frac{\partial^{2} F}{\partial \mathbf{u}^{2}}, \dot{\mathbf{u}}, \dot{\mathbf{u}}\right\rangle+2\left\langle\frac{\partial^{2} F}{\partial \mathbf{p} \partial \mathbf{u}}, \dot{\mathbf{p}}, \dot{\mathbf{u}}\right\rangle,
$$

the chain rule allows to expand $(6.2$ as follows:

$$
\begin{array}{r}
\mathbb{N}_{\mathbf{p}}^{\top} \mathbb{M} \mathbb{N}_{\mathbf{p}} \ddot{\mathbf{p}}+\mathbb{N}_{\mathbf{p}}^{\top} \mathbb{M}\left\langle\mathbf{D}^{2} F, \dot{\mathbf{p}}, \dot{\mathbf{u}}\right\rangle+\mathbb{N}_{\mathbf{p}}^{\top}\left\langle\Gamma(F(\mathbf{p}, \mathbf{u})), \mathbb{N}_{\mathbf{p}} \dot{\mathbf{p}}+\mathbb{N}_{\mathbf{u}} \dot{\mathbf{u}}, \mathbb{N}_{\mathbf{p}} \dot{\mathbf{p}}+\mathbb{N}_{\mathbf{u}} \dot{\mathbf{u}}\right\rangle \\
+\mathbb{N}_{\mathbf{p}}^{\top} \mathbb{M}_{\mathbf{u}} \ddot{\mathbf{u}}+\mathbb{N}_{\mathbf{p}}^{\top} \frac{\partial P^{\top}}{\partial \mathbf{q}}+\mathbb{N}_{\mathbf{p}}^{\top} \frac{\partial V^{\top}}{\partial \mathbf{q}}=\mathbf{0}, \quad(t \geq 0) .
\end{array}
$$

The function $F$ being given, the matrices $\mathbb{N}_{\mathbf{p}}$ and $\mathbb{N}_{\mathbf{u}}$ and the tensor $\mathbf{D}^{2} F$ are explicitly known. We know how to evaluate all of the terms in (5.1) and therefore we draw the same conclusion for $(6.3)$. Some care has to be taken when choosing the function $F$ for the ODE (6.3) to be well posed. Let us consider a given control $\mathbf{u}: \mathbb{R}_{+} \rightarrow \mathcal{U}$.

Theorem 6.1 Assume that $t \in \mathbb{R}_{+} \mapsto \mathbf{u} \in \mathcal{U}$ is of classe $C^{2,1}$ (respectively $C^{2+k}$ with $k \geq 1$ or analytic) and assume that $\mathbb{N}_{\mathbf{p}}$ is full rank for all $(\mathbf{p}, \mathbf{u}) \in$ $\mathcal{P} \times \mathcal{U}$. Then, for any initial data $\left(\mathbf{p}_{0}, \dot{\mathbf{p}}_{0}\right) \in T \mathcal{P}$ there exists a unique solution $t \in[0, T) \mapsto \mathbf{p} \in \mathcal{P}$ to $O D E$ (6.3) such that $\left(\mathbf{p}(0), \frac{d \mathbf{p}}{d t}(0)\right)=\left(\mathbf{p}_{0}, \dot{\mathbf{p}}_{0}\right)$.

Further, the solution if of class $C^{2}$ (respectively $C^{2+k}$ or analytic) and either $T=+\infty$ or $T$ corresponds to the time of a collision between two articulated bodies or between a body with any boundary of the fluid domain.

The hypothesis on the rank of $\mathbb{N}_{\mathbf{p}}$ is not surprising. It means that for any value of the control variable $\mathbf{u}$, the function $\mathbf{p} \mapsto F(\mathbf{p}, \mathbf{u})$ is locally an immersion from $\mathcal{P}$ into $\mathcal{Q}$.

Proof: We use the same arguments as in the proof of Theorem 4.1.

The hypothesis on $\mathbb{N}_{\mathbf{p}}$ ensures that the matrix $\mathbb{N}_{\mathbf{p}}^{\top} \mathbb{M}_{\mathbf{p}}$ is positive definite for all $(\mathbf{p}, \mathbf{u})$ and hence always invertible.

From the definition 6.1), we deduce that:

$$
\mathcal{L}:=\frac{1}{2} \dot{\mathbf{p}}^{\top} \mathbb{N}_{\mathbf{p}}^{\top} \mathbb{M N}_{\mathbf{p}} \dot{\mathbf{p}}+\dot{\mathbf{p}}^{\top} \mathbb{N}_{\mathbf{p}}^{\top} \mathbb{M N}_{\mathbf{u}} \dot{\mathbf{u}}+\frac{1}{2} \dot{\mathbf{u}}^{\top} \mathbb{N}_{\mathbf{u}}^{\top} \mathbb{M N}_{\mathbf{u}} \dot{\mathbf{u}}-P(F(\mathbf{p}, \mathbf{u}))
$$

We set then:

$$
P^{*}:=-\frac{1}{2} \dot{\mathbf{u}}^{\top} \mathbb{N}_{\mathbf{u}}^{\top} \mathbb{M N}_{\mathbf{u}} \dot{\mathbf{u}}+P(F(\mathbf{p}, \mathbf{u})),
$$


and we introduce the energy like amount:

$$
E^{*}:=\frac{1}{2} \dot{\mathbf{p}}^{\top} \mathbb{N}_{\mathbf{p}}^{\top} \mathbb{M} \mathbb{N}_{\mathbf{p}} \dot{\mathbf{p}}+P^{*} .
$$

Proceeding like in the proof of Theorem 4.1, we prove that $E^{*}$ remains constant for all time and hence that the solution cannot blow up until a collision occurs.

\section{Computation of internal forces and torques}

Rather than $\mathbf{u}$ governing the shape-changes of the bodies, a more realistic control variable would be the internal forces and torques causing these shape-changes. Actually, under an additional assumption on $F$, we will show that both choices are equivalent by proving that there is a one-to-one relation between $\mathbf{u}$ and the internal forces.

First, the generalized forces causing the shape-changes driven by $\mathbf{u}$ are defined in Lagrangian Mechanics as the right hand side $\boldsymbol{\nu}$ in the equality:

$$
\frac{d}{d t} \frac{\partial \mathcal{L}}{\partial \dot{\mathbf{u}}}-\frac{\partial \mathcal{L}}{\partial \mathbf{u}}=\boldsymbol{\nu}, \quad(t \geq 0) .
$$

We can easily expand this identity by copying-pasting $(6.3)$ and exchanging $\mathbf{u}$ and $\mathbf{p}$ for they play symmetrical roles:

$$
\begin{array}{r}
\mathbb{N}_{\mathbf{u}}^{\top} \mathbb{M N}_{\mathbf{u}} \ddot{\mathbf{u}}+\mathbb{N}_{\mathbf{u}}^{\top} \mathbb{M}\left\langle\mathbf{D}^{2} F, \dot{\mathbf{p}}, \dot{\mathbf{u}}\right\rangle+\mathbb{N}_{\mathbf{u}}^{\top}\left\langle\Gamma(F(\mathbf{p}, \mathbf{u})), \mathbb{N}_{\mathbf{p}} \dot{\mathbf{p}}+\mathbb{N}_{\mathbf{u}} \dot{\mathbf{u}}, \mathbb{N}_{\mathbf{p}} \dot{\mathbf{p}}+\mathbb{N}_{\mathbf{u}} \dot{\mathbf{u}}\right\rangle \\
+\mathbb{N}_{\mathbf{u}}^{\top} \mathbb{M N}_{\mathbf{p}} \ddot{\mathbf{p}}+\mathbb{N}_{\mathbf{u}}^{\top} \frac{\partial P^{\top}}{\partial \mathbf{q}}+\mathbb{N}_{\mathbf{u}}^{\top} \frac{\partial V^{\top}}{\partial \mathbf{q}}=\boldsymbol{\nu}, \quad(t \geq 0) .
\end{array}
$$

Therefore, for any given smooth control $t \in \mathbb{R}_{+} \mapsto \mathbf{u} \in \mathcal{U}$, we can compute first $\mathbf{p}$ by solving 6.3 and next use it to compute $\boldsymbol{\nu}$ with 7.2 . The converse is true if the system of equations (6.3)- (7.2), seen as a system of ODEs in $(\mathbf{p}, \mathbf{u})$ can be solved for any given right hand side $\boldsymbol{\nu}$. Indeed, let now the function $t \in \mathbb{R}_{+} \mapsto \boldsymbol{\nu} \in \mathbb{R}^{d}$ be given.

Theorem 7.1 Assume that both $\mathbb{N}_{\mathbf{p}}$ and $\mathbb{N}_{\mathbf{u}}$ are full-rank for all $(\mathbf{p}, \mathbf{u}) \in \mathcal{P} \times \mathcal{U}$ and that the function $\boldsymbol{\nu}$ is Lipschitz continuous (respectively of class $C^{k}$ with $k \geq 1$ or analytic). Then, for any $\left(\mathbf{p}_{0}, \dot{\mathbf{p}}_{0}, \mathbf{u}_{0}, \dot{\mathbf{u}}_{0}\right) \in T \mathcal{P} \times T \mathcal{U}$, there exists a unique solution $t \in[0, T) \mapsto(\mathbf{p}, \mathbf{u}) \in \mathcal{P} \times \mathcal{U}$ to the system of ODEs (6.3)-(7.2) such that $\left(\mathbf{p}(0), \frac{d \mathbf{p}}{d t}(0), \mathbf{u}(0), \frac{d \mathbf{u}}{d t}(0)\right)=\left(\mathbf{p}_{0}, \dot{\mathbf{p}}_{0}, \mathbf{u}_{0}, \dot{\mathbf{u}}_{0}\right)$.

Further, the solution if of class $C^{2}$ (respectively $C^{2+k}$ or analytic) and either $T=+\infty$ or $T$ corresponds to the time of a collision between two articulated bodies or between a body with any boundary of the fluid domain.

The hypotheses on $\mathbb{N}_{\mathbf{p}}$ and $\mathbb{N}_{\mathbf{u}}$ entail that $F$ is a local diffeomorphism from $\mathcal{P} \times \mathcal{U}$ onto $\mathcal{Q}$. 
Proof: Once more, we proceed like in the proof of Theorem 6.1 and we obtain the existence of a solution $t \mapsto(\mathbf{p}, \mathbf{u}) \in \mathcal{P} \times \mathcal{U}$ local in time. We denote by $T$ the maximal time of existence of the solution, we assume that $T<+\infty$ and we define the natural overall energy by:

$$
E:=\frac{1}{2} \dot{\mathbf{p}}^{\top} \mathbb{N}_{\mathbf{p}}^{\top} \mathbb{M} \mathbb{N}_{\mathbf{p}} \dot{\mathbf{p}}+\dot{\mathbf{p}}^{\top} \mathbb{N}_{\mathbf{p}}^{\top} \mathbb{M N}_{\mathbf{u}} \dot{\mathbf{u}}+\frac{1}{2} \dot{\mathbf{u}}^{\top} \mathbb{N}_{\mathbf{u}}^{\top} \mathbb{M N}_{\mathbf{u}} \dot{\mathbf{u}}+P(F(\mathbf{p}, \mathbf{u})) .
$$

We next easily check that:

$$
\frac{\partial \mathcal{L}}{\partial \dot{\mathbf{p}}} \cdot \dot{\mathbf{p}}+\frac{\partial \mathcal{L}}{\partial \dot{\mathbf{u}}} \cdot \dot{\mathbf{u}}-\mathcal{L}=E .
$$

Differentiating this equality with respect to time, we get:

$$
\left(\frac{d}{d t} \frac{\partial \mathcal{L}}{\partial \dot{\mathbf{u}}}-\frac{\partial \mathcal{L}}{\partial \mathbf{u}}\right) \cdot \dot{\mathbf{u}}=\frac{d E}{d t},
$$

meaning that the variation of energy is equal to the power-like amount $\boldsymbol{\nu} \cdot \dot{\mathbf{u}}$ expended by the internal forces. Assume now that $F(\mathbf{p}, \mathbf{u})$ remains in a compact subset $\mathcal{K}$ of $\mathcal{Q}$ for all $t \in\left[0, T\right.$. Then, there exist $\alpha>0$ and $P_{\min } \in \mathbb{R}$ (the minimum of the potential energy $P$ in $\mathcal{K})$ such that, for all $(\mathbf{p}, \mathbf{u}) \in F^{-1}(\mathcal{K})$ :

$$
\alpha|\dot{\mathbf{u}}|^{2} \leq E-P_{\min } .
$$

According to 27.3$]$, we deduce that for all $t \in[0, T[$ :

$$
\alpha|\dot{\mathbf{u}}|^{2} \leq \lambda+\int_{0}^{t}|\boldsymbol{\nu}||\dot{\mathbf{u}}| \mathrm{d} s
$$

where $\lambda:=\left|E(0)-P_{\min }\right|$. Setting then:

$$
\phi(t):=\int_{0}^{t}|\boldsymbol{\nu} \| \dot{\mathbf{u}}| \mathrm{d} s,
$$

we obtain after some basic algebra, that for all $t \in[0, T[$ :

$$
\frac{\phi^{\prime}(t)}{\sqrt{\phi(t)+\lambda}} \leq \frac{|\boldsymbol{\nu}|}{\sqrt{\alpha}}
$$

Integrating this inequality with respect to time, we get the estimate:

$$
\phi(t) \leq \lambda+\left[\sqrt{\lambda}+\frac{1}{2 \sqrt{\alpha}} \int_{0}^{t}|\boldsymbol{\nu}| \mathrm{d} s\right]^{2} .
$$

After plugging this result into 7.4 , we just have proved the Gronwall-type inequality:

$$
|\dot{\mathbf{u}}|^{2} \leq \frac{2}{\alpha} \lambda+\frac{1}{\alpha}\left[\sqrt{\lambda}+\frac{1}{2 \sqrt{\alpha}} \int_{0}^{t}|\boldsymbol{\nu}| \mathrm{d} s\right]^{2},
$$


meaning that $\dot{\mathbf{u}}$ is bounded for all $t \in[0, T$ [. Seeing $(7.3)$, this entails that $E$ is also bounded and hence that $\dot{\mathbf{p}}$ is bounded as well. Classical behavior results for solutions of ODEs tell us that this result contradicts the hypothesis $T<+\infty$. We deduce that either $T=+\infty$ or

$$
\lim _{t \rightarrow T} F(\mathbf{p}, \mathbf{u}) \in \partial \mathcal{Q},
$$

what means a collision in our system at the time $T$.

One of the interest in computing the internal forces and torques is that it allows to quantify the efficiency of a strategy of locomotion. Indeed, a relevant cost functional associated with a displacement over a time interval $[0, T]$ could be:

$$
\int_{0}^{\top}|\boldsymbol{\nu}|^{2} \mathrm{~d} s .
$$

This approach was for instance adopted in [12] to seek optimal strokes.

\section{Numerical scheme}

\subsection{Introduction}

The equations governing the motion of a collection of solids or articulated bodies are ODEs. Therefore our numerical scheme will involve an ODE solver. Consider for instance the ODE (5.1) without the circulation term (circulation has not been implemented yet) and let us turn it into the normal form:

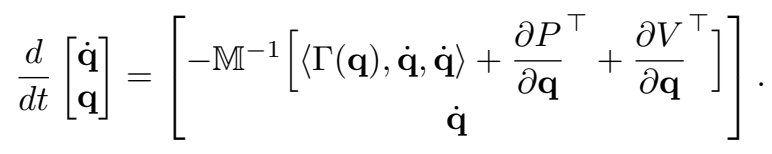

Any evaluation of the right hand side requires the computation of the elementary potential functions and hence our numerical scheme will also involve a solver of boundary value problems.

Related to the potential functions, the amounts we need to compute are more precisely the elements of the mass matrices:

$$
\int_{\mathcal{F}} \nabla \psi_{k}^{i} \cdot \nabla \psi_{l}^{j} \mathrm{~d} \mathbf{x},
$$

for all $i, j \in\{1, \ldots, n\}$ and $k, l \in\{1,2,3\}$ as well as their derivatives with respect to q. As already mentioned before, all of these quantities can be expressed as integrals on the boundaries of the solids (see formulae (2.15) and (2.11)). This is also true for the repulsive force $\partial V / \partial \mathbf{q}$ (see (5.2)).

Conversely, all of the elementary potential functions, as being solutions of Neumann boundary value problems (abbreviated NBVP in the following), are completely determined by their (Neumann) data on the boundaries of the solids 
(see Appendix, Section A. Based on these considerations, our method will involve only computations on $\partial \mathcal{F}$ by means of an integral formulation. It is a fundamental point because one of the difficulty in dealing with fluid-structure problems is that the fluid domain depends on the unknown positions of the immersed structures. When the method involves computations in this domain, it needs to be meshed and the mesh has to be updated at each time step. The integral formulation allows this problem to be avoided. Another advantage of this approach is that the discretization of an integral equation set on the fluid boundary leads to a linear system having many less unknowns than the discretization of the same problem set in the fluid domain. At last, when the fluid domain is not bounded, dealing with integral equations allows to avoid the delicate problem of truncating the domain and determining artificial boundary conditions.

How to turn boundary value problems 2.6 into integral equations is a classical process (see [24]). For our problem, we have two choices for the integral formulation: the direct or the indirect type. The direct formulation best fits the problem requirements because it gives directly the Dirichlet boundary data as function of the Neumann boundary data, that is, corresponds to the Neumannto-Dirichlet operator. It reads (see [3]) for all $i \in\{1, \ldots, n\}, k \in\{1,2,3\}$ and $\mathbf{x} \in \partial \mathcal{F}:$

$$
\psi_{k}^{i}(\mathbf{x})-\frac{1}{\pi} \int_{\partial \mathcal{F}} \frac{\left(\mathbf{x}^{\prime}-\mathbf{x}\right)}{\left|\mathbf{x}^{\prime}-\mathbf{x}\right|^{2}} \cdot \mathbf{n}\left(\mathbf{x}^{\prime}\right) \psi_{k}^{i}\left(\mathbf{x}^{\prime}\right) \mathrm{d} \sigma_{\mathbf{x}^{\prime}}=-\frac{1}{\pi} \int_{\partial \mathcal{S}^{i}} \ln \left|\mathbf{x}^{\prime}-\mathbf{x}\right| b_{k}^{i}\left(\mathbf{x}^{\prime}\right) \mathrm{d} \sigma_{\mathbf{x}^{\prime}}
$$

where we recall that $b_{k}^{i}:=\mathbf{w}_{k}^{i} \cdot \mathbf{n}$ is the Neumann boundary data of $\psi_{k}^{i}$ and $\mathbf{n}$, the unitary normal to $\partial \mathcal{F}$, is directed toward the exterior of the fluid. An additionnal equation is needed when $\mathcal{F}$ is bounded:

$$
\int_{\partial \mathcal{S}} \psi_{k}^{i}\left(\mathbf{x}^{\prime}\right) \mathrm{d} \sigma_{\mathbf{x}^{\prime}}=0
$$

The next step consists in choosing a numerical method to solve 8.2 (coupled with 8.3 in the bounded case). In most of the articles dealing with the locomotion of shape-changing bodies in an ideal fluid, the authors use the so-called panel method [12, 13, 23. It is a collocation method where the approximation function space consists in piecewise constant functions built from a discretization of the boundary in small curved or straight segments of length $O(h)$. As a result, such a method is of order 1 , that is the error between the exact solution $\psi$ and the approximate one $\psi_{h}$ satisfies $\left\|\psi_{h}-\psi\right\|_{\infty}=O(h)$ with possibly superconvergence property at the collocation points $\xi_{k}:\left|\psi_{h}\left(\xi_{k}\right)-\psi\left(\xi_{k}\right)\right|=O\left(h^{2}\right)$ (see [9, remark 4.4 .8 page 85$]$ ).

For integral equations set on boundaries regular enough (described by parameterization), several authors [3, 7] advocate the Nyström method as the best possible choice because it is nearly as simple to implement as the panel method but has far better convergence properties. In the following we describe this method applied to our NVBP (see [3] for additional details). 


\subsection{Carrying out the Nyström method}

The accuracy of the Nyström method depends on the quadrature rule choosen to evaluate the integrals. In our case, all of the functions are defined on the fluid boundaries and hence, introducing parameterizations, can be seen as $2 \pi$-periodic functions defined on the interval $[0,2 \pi[$. For such functions, the rectangle rule is very well adapted as proved in [16, Corollary 9.27 page 210].

For all $i \in\{1, \ldots, n\}$, we introduce a twice continuously differentiable parameterization

$$
\gamma_{i}: s \in\left[0,2 \pi\left[\mapsto \gamma_{i}(s) \in \mathbb{R}^{2},\right.\right.
$$

of the boundary of $\mathcal{S}^{i}$. These parameterizations of the moving solids are obtained merely by composing given parameterizations of the fixed domains $\mathcal{S}_{0}^{i}$ with the rigid displacements $g^{i}:=\left(R^{i}, \mathbf{r}^{i}\right)$. We denote as well $\gamma_{i}(i \in\{n+$ $1, \ldots, N\})$ a parameterization of the remaining $N-n$ fixed fluid boundaries not shared with moving solids.

We define next for all $j, l \in\{1, \ldots, N\}$ the kernels $K_{j}^{l}$ and $R_{j}^{l}$ by:

$$
\begin{aligned}
K_{j}^{l}(t, s) & :=\frac{\left(\gamma_{j}(s)-\gamma_{l}(t)\right)}{\left|\gamma_{j}(s)-\gamma_{l}(t)\right|^{2}} \cdot \mathbf{n}\left(\gamma_{j}(s)\right)\left|\gamma_{j}^{\prime}(s)\right|, \\
R_{j}^{l}(t, s) & :=\ln \left|\gamma_{j}(s)-\gamma_{l}(t)\right|\left|\gamma_{j}^{\prime}(s)\right|, \quad(s, t \in[0,2 \pi[),
\end{aligned}
$$

and we rewrite 8.2 for all $i \in\{1, \ldots, n\}$ and $k \in\{1,2,3\}$ :

$$
\begin{aligned}
\psi_{k}^{i}\left(\gamma_{l}(t)\right)-\frac{1}{\pi} \sum_{j=1}^{N} \int_{0}^{2 \pi} & K_{j}^{l}(t, s) \psi_{k}^{i}\left(\gamma_{j}(s)\right) \mathrm{d} s \\
& =-\frac{1}{\pi} \int_{0}^{2 \pi} R_{i}^{l}(t, s) b_{k}^{i}\left(\gamma_{i}(s)\right) \mathrm{d} s, \quad(l=1, \ldots, N) .
\end{aligned}
$$

For any pair of indices $(i, k) \in\{1, \ldots, n\} \times\{1,2,3\}$, it is a linear system of $N$ integral equations with $N$ unknowns that are the parts of the solution $\psi_{k}^{i}$ living on each one of the $N$ boundaries. The kernels $K_{l}^{l}(t, s)$ have no singularity at $t=s$ since:

$$
\lim _{s \rightarrow t} K_{l}^{l}(t, s)=\frac{1}{2} \kappa\left(\gamma_{l}(t)\right)\left|\gamma_{l}^{\prime}(t)\right|
$$

where we recall that $\kappa$ is the curvature of the boundary.

Notation: The process we are going to describe being similar for any pair of indices $(i, k)$, let us assume that their are fixed. It allows us to drop them in the notation when possible and for instance merely denote $\psi$ for $\psi_{k}^{i}$.

This first stage done, we can now apply the Nyström method which is decomposed into two steps:

step 1 The domain $\left[0,2 \pi\right.$ [ of each parameterization $\gamma_{j}(j \in\{1, \ldots, N\})$ is uniformly discretized with $m_{j}:=2 n_{j}+1$ points $\left(n_{j} \geq 1\right)$ denoted $s_{p}^{j}:=$ 
$2 \pi p / m_{j}\left(p=0, \ldots, m_{j}-1\right)$. The integrals in the left hand side of 8.4$)$ are approximated for all $j, l \in\{1, \ldots, N\}$ using the left rectangular quadrature rule:

$$
\int_{0}^{2 \pi} K_{j}^{l}(t, s) \psi\left(\gamma_{j}(s)\right) \mathrm{d} s \simeq \frac{2 \pi}{m_{j}} \sum_{p=0}^{m_{j}-1} K_{j}^{l}\left(t, s_{p}^{j}\right) \psi\left(\gamma_{j}\left(s_{p}^{j}\right)\right), \quad(t \in[0,2 \pi[),
$$

which is quite accurate because, as already mentioned, $s \mapsto K_{j}^{l}(t, s) \psi\left(\gamma_{j}(s)\right)$ is a smooth $2 \pi$-periodic function. The same quadrature rule is used for the right hand side of 8.4 except for $l=i$ because the kernel $R_{i}^{i}(t, s)$ has an (integrable) singularity at $t=s$. This last problem is overcome using the decomposition ([3, page 329]) for any $(s, t) \in[0,2 \pi[\times[0,2 \pi[$ :

$$
\ln \left|\gamma_{i}(t)-\gamma_{i}(s)\right|=\ln \left|2 e^{-1 / 2} \sin \left(\frac{t-s}{2}\right)\right|+B^{i}(t, s),
$$

where:

$$
B^{i}(t, s):= \begin{cases}\ln \frac{\left|e^{1 / 2}\left(\gamma_{i}(t)-\gamma_{i}(s)\right)\right|}{|2 \sin ((t-s) / 2)|}, & \text { if } s \neq t, \\ \ln \left|e^{1 / 2} \gamma_{i}^{\prime}(t)\right| & \text { if } s=t .\end{cases}
$$

The function $B^{i}(t, s)$ is again a smooth periodic kernel with no singularity at $s=t$ and the corresponding integral is approximated using the rectangular rule with $m_{i}=2 n_{i}+1$ points. For the singular part, we use the formula below with with $\varphi(s):=\left|\gamma_{i}^{\prime}(s)\right| b_{k}^{i}\left(\gamma_{i}(s)\right) \mid$ :

$$
\begin{aligned}
&-\frac{1}{\pi} \int_{0}^{2 \pi} \varphi(s) \ln \left|2 e^{-1 / 2} \sin \left(\frac{t-s}{2}\right)\right| \mathrm{d} s= \\
& \frac{1}{\sqrt{2 \pi}}\left[\widehat{\varphi}_{0}+\sum_{|p|>0} \frac{\widehat{\varphi}_{p}}{|p|} e^{i p t}\right],
\end{aligned}
$$

available for any $2 \pi$-periodic smooth function $\varphi$ defined on $[0,2 \pi[$. The approximation of the right hand side of $8.5 \mathrm{a}$ is next done by

- truncating the series at $p= \pm n_{i}$,

- replacing the Fourier coefficients $\widehat{\varphi}_{p}\left(|p| \leq n_{i}\right)$ by their discrete Fourier transforms $\widehat{\Phi}_{p}$ (based on the same rectangular quadrature rule using the $m_{i}=1+2 n_{i}$ points $s_{j}^{i}$ of $[0,2 \pi[)$.

This can be written as the following matrix vector product:

$$
\widehat{\Phi}=\frac{\sqrt{2 \pi}}{m_{i}} \mathbb{F}_{m_{i}} \Phi, \quad(i=1, \ldots, n),
$$

where:

$$
\begin{aligned}
& \Phi:=\left[\varphi\left(s_{0}^{i}\right), \varphi\left(s_{1}^{i}\right), \ldots, \varphi\left(s_{m_{i}-1}^{i}\right)\right]^{\top}, \\
& \widehat{\Phi}:=\left[\widehat{\Phi}_{0}, \ldots, \widehat{\Phi}_{n_{i}}, \widehat{\Phi}_{-n_{i}}, \ldots, \widehat{\Phi}_{-1}\right]^{\top},
\end{aligned}
$$


and $\mathbb{F}_{m}$ is defined for any $m \geq 1$ as the usual discrete Fourier matrix:

$$
\mathbb{F}_{m}=\left[e^{-\frac{2 i \pi k j}{m}}\right]_{0 \leq k, j \leq m-1} .
$$

Denoting by $\widetilde{\psi}_{l}(t)$ and $\widetilde{y}_{l}(t)(l \in\{1, \ldots, N\})$ the approximations likewise obtained for respectively $\psi\left(\gamma_{l}(t)\right)$ and for the right hand side of $(8.4)$, the $N$ discretized equations (8.4) read:

$$
\widetilde{\psi}_{l}(t)-\frac{2}{m_{j}} \sum_{j=1}^{N} \sum_{p=0}^{m_{j}-1} K_{j}^{l}\left(t, s_{p}^{j}\right) \widetilde{\psi}_{j}\left(s_{p}^{j}\right)=\widetilde{y}_{l}(t), \quad(l=1, \ldots N) .
$$

Observe that once the quantities $\widetilde{\psi}_{j}\left(s_{p}^{j}\right)$ are known, this equation allows to define the numerical solution $\widetilde{\psi}=\left[\widetilde{\psi}_{1}, \ldots, \widetilde{\psi}_{N}\right]$ everywhere on the boundary. It is the so-called Nyström interpolation formula.

step 2 Finally the $N$ equations $(8.6)$ are turned into an equivalent linear square system in the unknowns $\psi_{j}\left(s_{p}^{j}\right)$ by requiring identities $(8.6)$ to be exact for $t=s_{p}^{l}\left(p=0, \ldots, m_{l}-1\right)$. We get then:

$$
\sum_{j=1}^{N} \mathbb{A}_{l, j} \Psi_{j}=Y_{l}, \quad(l=1, \ldots, N),
$$

where each block matrix $\mathbb{A}_{l, j}$ is of size $m_{l} \times m_{j}$. More precisely, we have:

$$
\begin{array}{rlrl}
{\left[\mathbb{A}_{\substack{l, j \\
l \neq j}}\right]_{q, p}} & =-\frac{2}{m_{j}} K_{j}^{l}\left(s_{q}^{l}, s_{p}^{j}\right), & & 0 \leq q \leq m_{l}-1, \quad 0 \leq p \leq m_{j}-1, \\
{\left[\mathbb{A}_{l, l}\right]_{q, p}} & =\delta_{q, p}-\frac{2}{m_{l}} K_{j}^{l}\left(s_{q}^{l}, s_{p}^{l}\right), & & 0 \leq q, p \leq m_{l}-1, \\
\Psi_{j} & =\left[\widetilde{\psi}_{j}\left(s_{0}^{j}\right), \ldots, \widetilde{\psi}_{j}\left(s_{m_{j}-1}^{j}\right)\right]^{\top}, & & \\
Y_{l} & =\left[\widetilde{y}_{l}\left(s_{0}^{l}\right), \ldots, \widetilde{y}_{l}\left(s_{m_{l}-1}^{l}\right)\right]^{\top}, & l=1, \ldots, N .
\end{array}
$$

As already mentioned, $Y_{l}$ for $l \neq i$ is built using the rectangular rule:

$$
\left[Y_{l}\right]_{q}=-\frac{2}{m_{i}} \sum_{p=0}^{m_{i}-1} R_{i}^{l}\left(s_{q}^{l}, s_{p}^{i}\right) b_{k}^{i}\left(\gamma_{i}\left(s_{p}^{i}\right)\right), \quad 0 \leq q \leq m_{l}-1 .
$$

For $l=i$, the special treatment consisting of process 8.5 leads to:

$$
\begin{aligned}
Y_{i} & =\mathbb{D} \Phi+\mathbb{F}_{m_{i}}^{-1} \operatorname{diag}(h) \mathbb{F}_{m_{i}} \Phi, \\
{[\mathbb{D}]_{q, p} } & =-\frac{2}{m_{i}} B^{i}\left(s_{q}^{i}, s_{p}^{i}\right), \quad 0 \leq q, p \leq m_{i}-1, \\
h & =\left[1,1,1 / 2, \ldots, 1 / n_{i}, 1 / n_{i}, \ldots, 1 / 2,1\right]^{\top},
\end{aligned}
$$


where the term $\mathbb{D} \Phi$ corresponds to the rectangular rule applied to the smooth kernel $B^{i}$. The other part is computed fastly in $O\left(\ln \left(m_{i}\right) m_{i}\right)$ operations using the fast Fourier transform algorithm followed by $m_{i}$ divisions and finally by the inverse fast Fourier transform (this reads simply ifft(h.*fft(Phi)) in Matlab language).

The overall matrix $\mathbb{A}$ related to the linear system 8.7 is defined by blocks:

$$
\mathbb{A}:=\left[\begin{array}{ccc}
\mathbb{A}_{1,1} & \ldots & \mathbb{A}_{1, n} \\
\vdots & & \vdots \\
\mathbb{A}_{n, 1} & \ldots & \mathbb{A}_{n, n}
\end{array}\right] .
$$

Unlike the right hand side term in system 8.7) which depends on the potential $\psi_{k}^{i}$ we wish to compute, the matrix $\mathbb{A}$ is the same for all of the elementary potentials.

The accuracy of this scheme (discussed in [3, page 333]) depends on the smoothness of both the Neumann boundary data and the boundaries parameterization. In our case the parameterization is of class $C^{\ell}(\ell \geq 2)$ while the Neumann data $b_{i}^{k}$ are $C^{\ell-1}$. So, according to [3, page 333], the approximate Nyström solution $\widetilde{\psi}_{i}^{k}$ satisfies, at least when $\mathcal{F}$ is not bounded:

$$
\left\|\widetilde{\psi}_{i}^{k}-\psi_{i}^{k}\right\|_{\infty}=O\left(\ln (h) h^{\ell-1}\right), \quad h:=\max _{i} \frac{2 \pi}{m_{i}} .
$$

When $\mathcal{F}$ is bounded the matrix $\mathbb{A}$ is not invertible any longer (or at least very badly conditioned). It is not surprising since the solution of the continuous problem is defined only up to a constant and needs for instance 8.3 to recover uniqueness. A way to solve this problem consists in replacing one of the equations by the linear equation resulting from the discretization of 8.3 :

$$
\sum_{l=1}^{n} \frac{2 \pi}{m_{l}} \sum_{p=0}^{m_{l}-1} \widetilde{\psi}_{k}^{i}\left(s_{p}^{l}\right)\left|\gamma_{i}^{\prime}\left(s_{p}^{l}\right)\right|=0 .
$$

For symmetry reasons, the removed equation is first substracted to all of the others before being replaced by 8.8 . Although we have not carried out a rigorous analysis, this choice has been preferred after several numerical tests to the same method but without substraction and to the classical method consisting in imposing a value at a given point.

\subsection{Additional details}

The linear system 8.7 must be solved for all of the elementary potentials $\psi_{k}^{i}$ $(i \in\{1, \ldots, n\}, k \in\{1,2,3\})$, that is to say for $3 n$ different right hand side terms built from the $3 n$ boundary data $b_{k}^{i}$. Since the matrix $\mathbb{A}$ remains unchanged, the more efficient way to carry out this task consists in using a $L U$ factorization rather than an iterative method especially when $n$ is large. 
Once the elementary potentials have been computed, a lot of contour integrals have to be approximated in 2.11) and 2.15. We use once again the rectangular formula with the same boundary discretization $\left(s_{p}^{j}=2 \pi p / m_{j}\right.$, $\left.p=0, \ldots, m_{j}-1\right)$.

Some terms in 2.11) and 2.15 require the knowledge at the integration points $s_{p}^{j}$ of the tangential derivatives of the potentials. This is done by trigonometric interpolation and so the discrete Fourier transform and its inverse are again involved.

Eventually, we have experienced several Matlab built-in ODEs solvers and have selected ode113 which is a variable order Adams-Bashforth-Moulton PECE solver (we refer to the Matlab documentation for details).

\section{Biohydrodynamics Matlab Toolbox}

\subsection{Introduction}

The Biohydrodynamics Toolbox (BhT) is a Matlab Toolbox allowing to realize numerical simulations of rigid solids immersed in a potential flow. The system fluid-solids can be either confined, partially bounded or unbounded. The buoyant force and collisions between solids are supported (but circulation is not yet). The solids can be free of constraints or linked together in order to constitute articulated bodies. In the latter case, the constraints are prescribed by the user as functions of time and the induced motion is computed. Post-processing functions are provided to compute the internal torques, the power and the energy expended by the swimming bodies.

BhT is free (distributed under license GNU GPL) and comes with complete html documentation including plenty examples and tutorials. It is available at:

- http://bht.gforge.inria.fr/ (hosted by INRIA Gforge).

- http://www .mathworks . com/matlabcentral/fileexchange/21872 (hosted by Matlab Central).

Going through all of the features of BhT would be too long and is out of the scope of this article. We will rather use BhT to discuss and illustrate Sparenberg's affirmation in [28, page 63] saying that: a body of finite extent, moving periodically through an inviscid and incompressible fluid without shedding vorticity, cannot exert a force with non-zero mean value. In particular, we will show that this sentence seems no longer true when the fluid domain contains fixed obtacles.

\subsection{Settings of the simulation}

We consider a fish-like swimming body made of four ellipse-shaped rigid solids as pictured in figure 2. The three angles of the joints are driven by the functions drawn in fig 3 over the time interval $[0,6 \pi]$. Notice that all of the functions are 


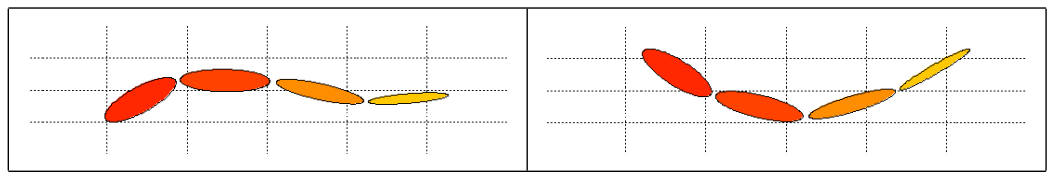

Figure 2: Screenshots of the swimming fish

compactly supported. The fish starts from rest, undergoes shape-changes yielding a net forward motion and stop back in its initial shape. We are interesting in studying its final velocity. Indeed, denoting by $m>0$ the total mass of the fish and by $\dot{\mathbf{r}}$ the velocity of its center of mass, Newton's laws tell us that:

$$
\frac{1}{6 \pi} m \dot{\mathbf{r}}(6 \pi)=\frac{1}{6 \pi} \int_{0}^{6 \pi} F \mathrm{~d} t
$$

where $F$ stands for the hydrodynamical forces generated by the fish to propel itself. Thereby, the right hand side in (9.1) is precisely the mean value of this force over the time interval $[0,6 \pi]$.

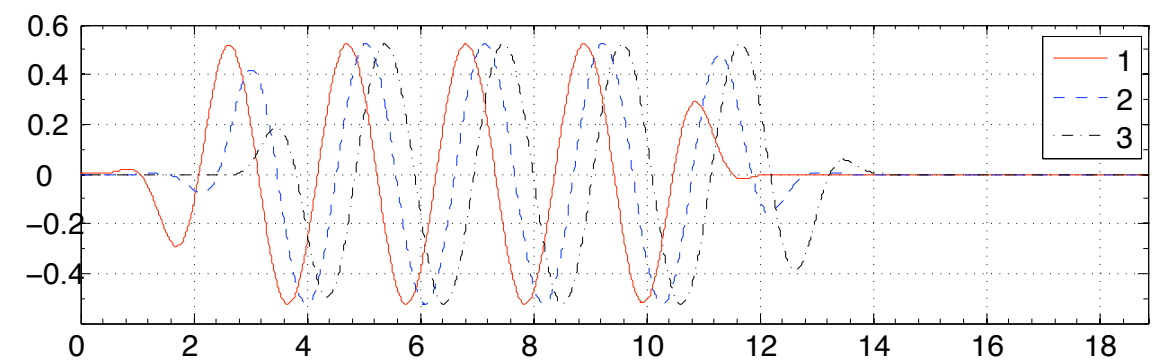

Figure 3: Angles of the joints with respect to time (given functions). Joint 1 corresponds to the fish's head and joint 3 to the tail.

We are going to perform twice the same simulation. In a first time, the fish will be alone in the fluid and the system fluid-fish will fill the whole space. In a second time, the fluid will contain also two fixed rectangular obstacles and the fish will pass through them in its course (see figure 4 ).

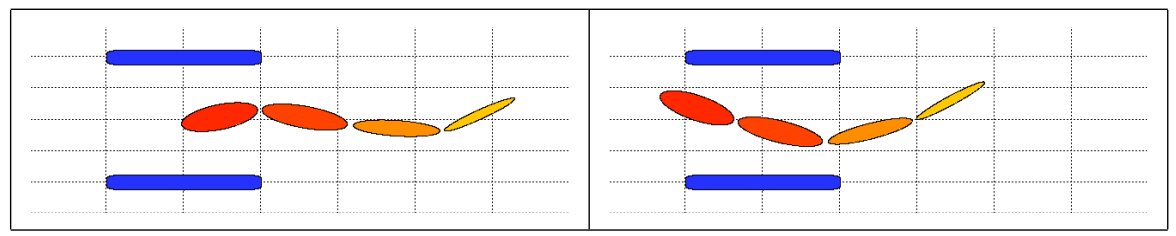

Figure 4: Fish swimming between two obstacles

The movies are available at:

http://bht.gforge.inria.fr/Examples/demos.html 


\subsection{Results}

With BhT we can compute the velocity of the center of mass of the fish at any time. It is pictured in figures 5 and 6 .

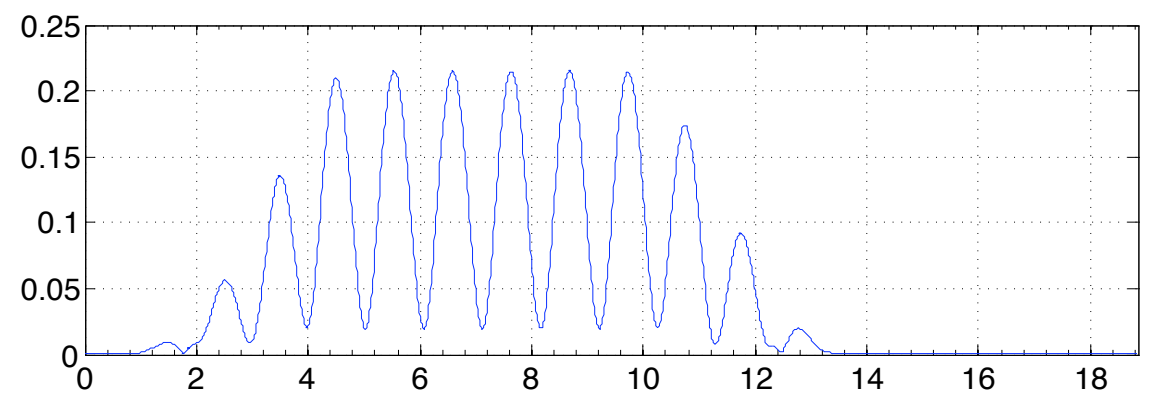

Figure 5: Velocity of the center of mass of the fish with respect to time (fluid without obstacle).

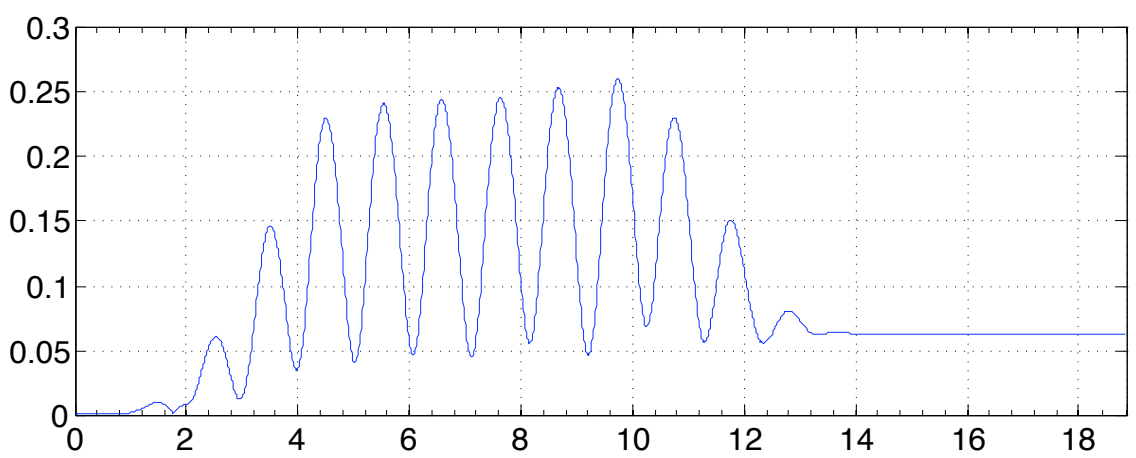

Figure 6: Velocity of the center of mass of the fish with respect to time (fluid with obstacles).

We clearly observe in figure 5 that the final velocity of the fish is zero, which confirms Sparenberg's statement in this case. On the other hand, with the same sequence of strokes, figure 6 tells us that the fish generates non zero mean thrust when the fluid contains obstacles.

An other interesting point that can be observed using BhT is that the torques applied at the joints to produce the shape-changes are not equal in both cases although the deformations are. In figure 7 we have drawn the values of the torques for the first case (fluid without obtacles) and in figure 8 the difference between the values of the torques in the first and second cases.

Computing next the cost of each displacement as quantified by the cost functional 7.5 , we obtain that the second strategy is $3.22 \%$ cheaper than the first one. These surprising results can be summarized by saying that not only 
generates the fish non-zero mean thrust by swimming through obstacles but also it swims more effortlessly.

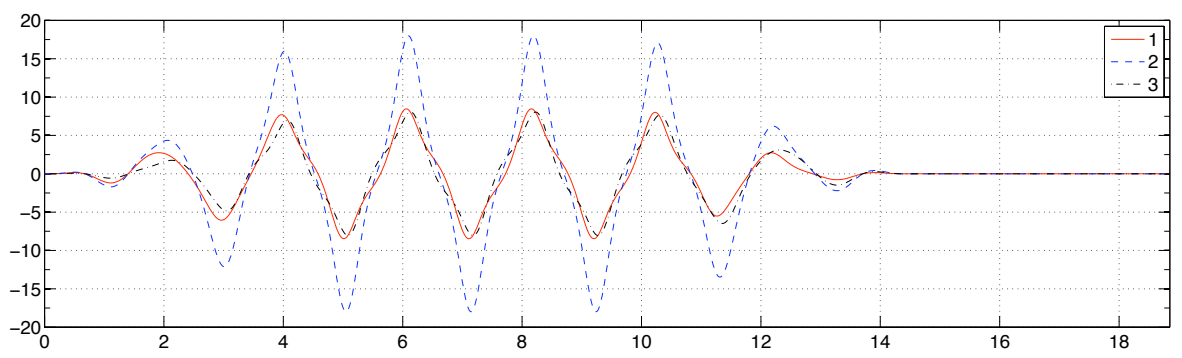

Figure 7: Values of the torques at the joints with respect to time in the first case.

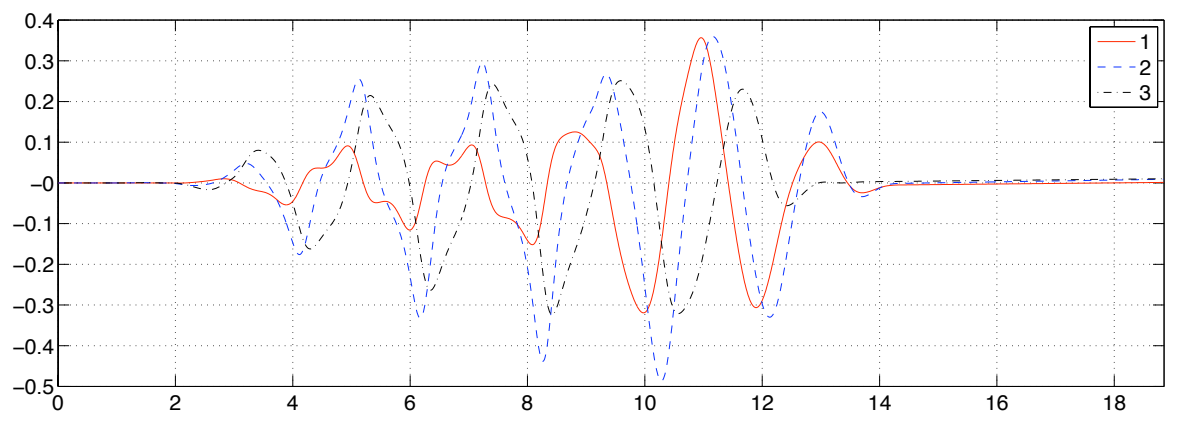

Figure 8: Difference between the values of the torques at the joints in the first and second case.

\section{A Neumann boundary value problem}

This section is dedicated to recalling some results stated in 4, 1, 2, about the well-posedness of Neumann boundary value problems. We write the problem in the generic form:

$$
\begin{aligned}
-\Delta \psi & =0 \text { in } \mathcal{F}, \\
\partial_{\mathbf{n}} \psi & =g \text { on } \partial \mathcal{F},
\end{aligned}
$$

and we assume that $g \in H^{-1 / 2}(\partial \mathcal{F})$ is given $\left(H^{-1 / 2}(\partial \mathcal{F})\right.$ is the dual space of the Sobolev space $H^{1 / 2}(\partial \mathcal{F})$; see [19, $\S 7.3$ pages 38-42]). For problem (A.1) to admit solutions, $g$ has to satisfy the compatibility condition:

$$
\langle g, 1\rangle_{H^{-1 / 2}(\partial \mathcal{F}) \times H^{1 / 2}(\partial \mathcal{F})}=0 .
$$




\section{A.1 Bounded domain}

In this case, we introduce the function space:

$$
X^{1}(\mathcal{F}):=\left\{\psi \in H^{1}(\mathcal{F}): \Delta \psi \in L^{2}(\mathcal{F})\right\} .
$$

For any $\psi \in X^{1}(\mathcal{F})$, its normal trace $\partial_{\mathbf{n}} \psi$ exists in $H^{-1 / 2}(\partial \mathcal{F})$. Lax-Milgram Theorem ensures that there exists a unique weak solution $\psi \in X^{1}(\mathcal{F})$ satisfying both identities:

$$
\begin{aligned}
\int_{\mathcal{F}} \nabla \psi \cdot \nabla \varphi \mathrm{d} \mathbf{x} & =\int_{\partial \mathcal{F}} g \varphi \mathrm{d} \sigma, \quad \forall \varphi \in H^{1}(\mathcal{F}), \\
\int_{\partial \mathcal{S}} \psi \mathrm{d} \sigma & =0 .
\end{aligned}
$$

Moreover, we have an estimate:

$$
\|\psi\|_{X^{1}(\mathcal{F})} \leq C\|g\|_{H^{-1 / 2}(\partial \mathcal{F})},
$$

for some constant $C>0$ independent of $\psi$ and $g$.

Assume now that $\partial \mathcal{F}$ is $C^{1,1}$ (continuously differentiable with first derivative Lipschitz continuous) and $g \in H^{1 / 2}(\partial \mathcal{F})$, then the solution $\psi$ of A.3 belongs to:

$$
X^{2}(\mathcal{F}):=\left\{\psi \in H^{2}(\mathcal{F}): \Delta\left(\partial_{x_{i}} \psi\right) \in L^{2}(\mathcal{F}), \forall i=1,2\right\} .
$$

For such function, both quantities $\partial_{\mathbf{n}}^{2} \psi:=\mathbf{n} \cdot D^{2} \psi \mathbf{n}$ and $\Delta_{\sigma} \psi$ (the LaplaceBeltrami operator) are well defined on $\partial \mathcal{F}$ as elements of $H^{-1 / 2}(\partial \mathcal{F})$. We have again an estimate:

$$
\|\psi\|_{X^{2}(\mathcal{F})} \leq C\|g\|_{H^{1 / 2}(\partial \mathcal{F})}
$$

for some constant $C>0$ independent of $\psi$ and $g$.

\section{A.2 Unbounded domain}

When $\mathcal{F}$ is not bounded, we define the weight function:

$$
\rho(\mathbf{x}):=\sqrt{1+|\mathbf{x}|^{2}} \log \left(2+|\mathbf{x}|^{2}\right),
$$

and the Lebesgue space:

$$
L_{N}^{2}(\mathcal{F}):=\left\{\psi \in \mathcal{D}^{\prime}(\mathcal{F}): \rho^{-1} \psi \in L^{2}(\mathcal{F})\right\}
$$

where $\mathcal{D}^{\prime}(\mathcal{F})$ is the space of distributions. Classical Sobolev spaces must be replaced by weighted Sobolev spaces:

$$
\begin{aligned}
& H_{N}^{1}(\mathcal{F}):=\left\{\psi \in L_{N}^{2}(\mathcal{F}): \partial_{x_{i}} \psi \in L^{2}(\mathcal{F}), \forall i=1,2\right\} \\
& X_{N}^{1}(\mathcal{F}):=\left\{\psi \in H_{N}^{1}(\mathcal{F}): \rho \Delta \psi \in L^{2}(\mathcal{F})\right\} \\
& H_{N}^{2}(\mathcal{F}):=\left\{\psi \in H_{N}^{1}(\mathcal{F}): \rho \partial_{x_{i} x_{j}}^{2} \psi \in L^{2}(\mathcal{F}), \forall i, j=1,2\right\}, \\
& X_{N}^{2}(\mathcal{F}):=\left\{\psi \in H_{N}^{2}(\mathcal{F}): \rho^{2} \Delta \psi \in L^{2}(\mathcal{F})\right\} .
\end{aligned}
$$

When $g \in H^{-1 / 2}(\partial \mathcal{F})$, the solution of A.3a A.3b exists in $H_{N}^{1}(\mathcal{F})$ and is unique. Further, it can be proved that the solution belongs to $X_{N}^{1}(\mathcal{F})$.

As in the case $\mathcal{F}$ bounded, if $\partial \mathcal{F}$ is $C^{1,1}$ and $g \in H^{1 / 2}(\partial \mathcal{F})$, then the solutions are in $X_{N}^{2}(\mathcal{F})$. 


\section{B Shape sensitivity analysis}

This section is self-contained and independent, including for the notation. We recall results of shape optimization theory, most of them being proved in [26].

Let $\mathcal{F}_{0} \subset \mathbb{R}^{2}$ and define for all $s=\left(s_{1}, \ldots, s_{n}\right) \in \mathbb{R}^{n}(n \geq 1)$ diffeomorphisms $\phi_{s}$ of class $C^{m}(m \geq 1)$ from $\mathbb{R}^{2}$ onto itself equal to the identity outside a large fixed compact ball $\mathcal{B}$. We denote by $\mathrm{D}_{m}$ the Banach space of such diffeomorphisms, endowed with the norm of the Sobolev space $W^{m, \infty}(\mathcal{B}, \mathcal{B})$ (uniform convergence in $\mathcal{B}$ of the function and the derivatives up to order $m$ ).

Denote $\mathcal{F}:=\phi_{s}\left(\mathcal{F}_{0}\right), \mathbf{w}_{i}:=\partial_{s_{i}} \phi_{s} \circ \phi_{s}^{-1}, \gamma_{i j}:=\partial_{s_{i} s_{j}}^{2} \phi_{s} \circ \phi_{s}^{-1}$ and $w_{i}^{\mathbf{n}}$ and $w_{i}^{\boldsymbol{\tau}}$ the normal and tangential components of $\mathbf{w}_{i}$ on $\partial \mathcal{F}$ such that:

$$
\mathbf{w}_{i}=w_{i}^{\mathbf{n}} \mathbf{n}+w_{i}^{\tau} \boldsymbol{\tau} .
$$

Define likewise $\gamma_{i j}^{\mathbf{n}}$, the normal component of $\gamma_{i j}$.

For any $i \in\{1, \ldots, n\}$, let then $\psi_{i}$ be the solution of the Neumann boundary value problem:

$$
\begin{aligned}
-\Delta \psi_{i} & =0 \quad \text { in } \mathcal{F}, \\
\partial_{\mathbf{n}} \psi_{i} & =w_{i}^{\mathbf{n}} \text { on } \partial \mathcal{F},
\end{aligned}
$$

and define for any $i, j \in\{1, \ldots, n\}$ the functional:

$$
J_{i j}(s):=\int_{\mathcal{F}} \nabla \psi_{i} \cdot \nabla \psi_{j} \mathrm{~d} \mathbf{x} .
$$

Under the general assumption that $\mathcal{F}_{0}$ is Lipschitz continuous, we have:

Proposition B.1 Assume that the mapping $s \mapsto \phi_{s} \in \mathrm{D}_{m}$ is of class $C^{k}$ with $k \geq 2$ (respectively analytic) and $m \geq 1$, then $s \mapsto J_{i j}$ is of class $C^{k-1}$ (respectively analytic).

The proof is given in 26] in a more general framework. Assuming additional regularity on $\partial \mathcal{F}_{0}$, we can compute the partial derivatives of $J_{i j}$ :

Proposition B.2 Assume that $\mathcal{F}_{0}$ is of class $C^{1,1}$ and that the mapping $s \mapsto$ $\phi_{s} \in \mathrm{D}_{m}$ is of class $C^{2}$ with $m \geq 2$. Then, for all $i, j, k \in\{1, \ldots, n\}$, we have:

$$
\begin{aligned}
& \frac{\partial}{\partial s_{i}}\left(\int_{\mathcal{F}} \nabla \psi_{j} \cdot \nabla \psi_{k} \mathrm{~d} \mathbf{x}\right)=-\int_{\partial \mathcal{F}}\left(\partial_{\boldsymbol{\tau}} \psi_{j} \partial_{\boldsymbol{\tau}} \psi_{k}\right) w_{i}^{\mathbf{n}} \mathrm{d} \sigma \\
& \quad-\int_{\partial \mathcal{F}}\left(\kappa \mathbf{w}_{i} \cdot \mathbf{w}_{j}-\gamma_{i j}^{\mathbf{n}}+\left(\partial_{\boldsymbol{\tau}} w_{j}^{\mathbf{n}} \cdot \boldsymbol{\tau}\right) w_{i}^{\boldsymbol{\tau}}+\left(\partial_{\boldsymbol{\tau}} w_{i}^{\mathbf{n}} \cdot \boldsymbol{\tau}\right) w_{j}^{\boldsymbol{\tau}}\right) \psi_{k} \mathrm{~d} \sigma \\
& \quad-\int_{\partial \mathcal{F}}\left(\kappa \mathbf{w}_{i} \cdot \mathbf{w}_{k}-\gamma_{i k}^{\mathbf{n}}+\left(\partial_{\boldsymbol{\tau}} w_{k}^{\mathbf{n}} \cdot \boldsymbol{\tau}\right) w_{i}^{\boldsymbol{\tau}}+\left(\partial_{\boldsymbol{\tau}} w_{i}^{\mathbf{n}} \cdot \boldsymbol{\tau}\right) w_{k}^{\boldsymbol{\tau}}\right) \psi_{j} \mathrm{~d} \sigma \\
& \quad+\int_{\partial \mathcal{F}} \partial_{\mathbf{n}} \psi_{i} \partial_{\mathbf{n}} \psi_{j} \partial_{\mathbf{n}} \psi_{k} \mathrm{~d} \sigma .
\end{aligned}
$$

Straightforward but tedious computations yield: 
Proposition B.3 Under the assumptions of Proposition B.2 and for all $i, j, k \in$ $\{1, \ldots, n\}$, the following identity holds:

$$
\begin{gathered}
\frac{\partial}{\partial s_{j}}\left(\int_{\partial \mathcal{F}} \psi_{i} \partial_{\mathbf{n}} \psi_{k} \mathrm{~d} \sigma\right)+\frac{\partial}{\partial s_{i}}\left(\int_{\partial \mathcal{F}} \psi_{j} \partial_{\mathbf{n}} \psi_{k} \mathrm{~d} \sigma\right)-\frac{\partial}{\partial s_{k}}\left(\int_{\partial \mathcal{F}} \psi_{i} \partial_{\mathbf{n}} \psi_{j} \mathrm{~d} \sigma\right)= \\
-\int_{\partial \mathcal{F}}\left(\partial_{\boldsymbol{\tau}} \psi_{k} \cdot \partial_{\boldsymbol{\tau}} \psi_{i}\right) w_{j}^{\mathbf{n}} \mathrm{d} \sigma-\int_{\partial \mathcal{F}}\left(\partial_{\boldsymbol{\tau}} \psi_{k} \cdot \partial_{\boldsymbol{\tau}} \psi_{j}\right) w_{i}^{\mathbf{n}} \mathrm{d} \sigma+\int_{\partial \mathcal{F}}\left(\partial_{\boldsymbol{\tau}} \psi_{i} \cdot \partial_{\boldsymbol{\tau}} \psi_{j}\right) w_{k}^{\mathbf{n}} \mathrm{d} \sigma \\
-2 \int_{\partial \mathcal{F}}\left(\kappa \mathbf{w}_{i} \cdot \mathbf{w}_{j}-\gamma_{i j}^{\mathbf{n}}+\left(\partial_{\boldsymbol{\tau}} w_{j}^{\mathbf{n}} \cdot \boldsymbol{\tau}\right) w_{i}^{\boldsymbol{\tau}}+\left(\partial_{\boldsymbol{\tau}} w_{i}^{\mathbf{n}} \cdot \boldsymbol{\tau}\right) w_{j}^{\boldsymbol{\tau}}\right) \psi_{k} \mathrm{~d} \sigma \\
+\int_{\partial \mathcal{F}} \partial_{\mathbf{n}} \psi_{k} \partial_{\mathbf{n}} \psi_{i} \partial_{\mathbf{n}} \psi_{j} \mathrm{~d} \sigma .
\end{gathered}
$$

\section{References}

[1] C. Amrouche, V. Girault, and J. Giroire. Weighted Sobolev spaces for Laplace's equation in $\mathbf{R}^{n}$. J. Math. Pures Appl. (9), 73(6):579-606, 1994.

[2] C. Amrouche, V. Girault, and J. Giroire. Dirichlet and Neumann exterior problems for the $n$-dimensional Laplace operator: an approach in weighted Sobolev spaces. J. Math. Pures Appl. (9), 76(1):55-81, 1997.

[3] K. E. Atkinson. The numerical solution of integral equations of the second kind, volume 4 of Cambridge Monographs on Applied and Computational Mathematics. Cambridge University Press, Cambridge, 1997.

[4] H. Brezis. Analyse fonctionnelle. Collection Mathématiques Appliquées pour la Maîtrise. [Collection of Applied Mathematics for the Master's Degree]. Masson, Paris, 1983.

[5] J. Carling, T. Williams, and G Bowtell. Self-propelled anguilliform swimming: simultaneous solution of the two-dimentional navier-stokes equations and newton's laws of motion. J. of Experimental Biology, 201:3143-3166, 1998.

[6] S. Childress. Mechanics of swimming and flying, volume 2 of Cambridge Studies in Mathematical Biology. Cambridge University Press, Cambridge, 1981.

[7] D. Colton and R. Kress. Inverse acoustic and electromagnetic scattering theory, volume 93 of Applied Mathematical Sciences. Springer-Verlag, Berlin, second edition, 1998.

[8] G. P. Galdi. On the steady self-propelled motion of a body in a viscous incompressible fluid. Arch. Ration. Mech. Anal., 148(1):53-88, 1999.

[9] W. Hackbusch. Integral equations, volume 120 of International Series of Numerical Mathematics. Birkhäuser Verlag, Basel, 1995. 
[10] A. Henrot and M. Pierre. Variation et optimisation de formes : une analyse géométrique, volume 048 of Mathématiques et applications. Springer, Berlin/Heidelberg/New York, 2005.

[11] J. Houot and A. Munnier. On the motion and collisions of rigid bodies in an ideal fluid. Asymptot. Anal., 56(3-4):125-158, 2008.

[12] E Kanso and J Marsden. Optimal motion of an articulated body in a perfect fluid. In in IEEE Conference on Decision and Control, pages 2511-2516, 2005 .

[13] E. Kanso, J. E. Marsden, C. W. Rowley, and J. B. Melli-Huber. Locomotion of articulated bodies in a perfect fluid. J. Nonlinear Sci., 15(4):255-289, 2005 .

[14] S. D. Kelly and R. M. Murray. Modelling efficient pisciform swimming for control. Internat. J. Robust Nonlinear Control, 10(4):217-241, 2000.

[15] V. V. Kozlov and D. A. Onishchenko. Motion of a body with undeformable shell and variable mass geometry in an unbounded perfect fluid. J. Dynam. Differential Equations, 15(2-3):553-570, 2003.

[16] R. Kress. Numerical analysis, volume 181 of Graduate Texts in Mathematics. Springer-Verlag, New York, 1998.

[17] H. Lamb. Hydrodynamics. Cambridge Mathematical Library. Cambridge University Press, Cambridge, sixth edition, 1993.

[18] J. Lighthill. Mathematical biofluiddynamics. Society for Industrial and Applied Mathematics, Philadelphia, Pa., 1975.

[19] J.-L. Lions and E. Magenes. Non-homogeneous boundary value problems and applications. Vol. I. Springer-Verlag, New York, 1972.

[20] H. Liu and K. Kawachi. A numerical study of undulatory swimming. J. comput. phys., 155(2):223-247, 1999.

[21] R. Mason and J. Burdick. Propulsion and control of deformable bodies in an ideal fluid. In Proceedings of the 1999 IEEE International Conference on Robotics and Automation, 1999.

[22] J. B. Melli, Rowley C. W., and D. S. Rufat. Motion planning for an articulated body in a perfect fluid. SIAM J. on Applied Dynamical Systems, 2006.

[23] Juan B. Melli, Clarence W. Rowley, and Dzhelil S. Rufat. Motion planning for an articulated body in a perfect planar fluid. SIAM J. Appl. Dyn. Syst., 5(4):650-669 (electronic), 2006. 
[24] S. G. Mikhlin. Mathematical physics, an advanced course. With appendices by V. M. Babič, V. G. Mazja and I. Ja. Bakelman. Translated from the Russian. North-Holland Series in Applied Mathematics and Mechanics, Vol. 11. North-Holland Publishing Co., Amsterdam, 1970.

[25] A. Munnier. Locomotion of deformable bodies in an ideal fluid: Newtonian versus lagrangian formalism. J. Nonlinear Sci., 2008.

[26] A. Munnier. On the self-displacement of deformable bodies in a potential fluid flow. Math. Models Methods Appl. Sci., 18(11):1945-1981, december 2008.

[27] J. San Martin, J. F. Scheid, T. Takahashi, and M. Tucsnak. An initial and boundary problem modeling fish-like swimming. Arch. Ration. Mech. Anal., 2008.

[28] J. A. Sparenberg. Hydrodynamic propulsion and its optimization (Analytic theory). Fluid Mechanics and its Applications. 27. Dordrecht: Kluwer Academic Publishers. 384 p., 1994.

[29] J. A. Sparenberg. Survey of the mathematical theory of fish locomotion. J. Engrg. Math., 44(4):395-448, 2002.

[30] G. Taylor. Analysis of the swimming of microscopic organisms. Proc. R. Soc. Lond., Ser. A, 209:447-461, 1951.

[31] G. Taylor. Analysis of the swimming of long and narrow animals. Proc. $R$. Soc. Lond., Ser. A, 214:158-183, 1952.

[32] M. S. Triantafyllou, G. S. Triantafyllou, and D. K. P. Yue. Hydrodynamics of fishlike swimming. In Annual review of fluid mechanics, Vol. 32, volume 32 of Annu. Rev. Fluid Mech., pages 33-53. Annual Reviews, Palo Alto, CA, 2000.

[33] T. Y. Wu. Mathematical biofluiddynamics and mechanophysiology of fish locomotion. Math. Methods Appl. Sci., 24(17-18):1541-1564, 2001.

[34] Q. Zhu, M. J. Wolfgang, D. K. P. Yue, and M. S. Triantafyllou. Threedimensional flow structures and vorticity control in fish-like swimming. $J$. Fluid Mech., 468:1-28, 2002. 